\title{
Philosophiques
}

\section{L’idéalisme britannique : histoire et actualité}

\section{Sébastien Gandon et Mathieu Marion}

Volume 36, numéro 1, printemps 2009

L’idéalisme britannique

URI : https://id.erudit.org/iderudit/038008ar

DOI : https://doi.org/10.7202/038008ar

Aller au sommaire du numéro

\section{Éditeur(s)}

Société de philosophie du Québec

ISSN

0316-2923 (imprimé)

1492-1391 (numérique)

Découvrir la revue

Citer cet article

Gandon, S. \& Marion, M. (2009). L'idéalisme britannique : histoire et actualité. Philosophiques, 36(1), 3-34. https://doi.org/10.7202/038008ar

\section{Résumé de l'article}

L'idéalisme britannique est un mouvement qui a dominé les universités britanniques (et celles de son empire) pendant une cinquantaine d'années à la fin $\mathrm{du} \mathrm{XIX}^{\mathrm{e}}$ siècle et au début du $\mathrm{XX}^{\mathrm{e}}$ siècle, mais qui est passé presque totalement inaperçu dans le monde francophone. Rejetés en bloc par les philosophes analytiques, ces auteurs ont aussi été ignorés pendant longtemps dans leur pays, mais certains d'entre eux, notamment Bradley et Collingwood, jouissent d'un regain d'intérêt à la faveur d'un renouveau des études sur les origines de la philosophie analytique. Ce texte est une introduction à l'idéalisme britannique, qui retrace, dans une première partie plus historique, les grandes lignes de sa genèse, son développement et son déclin. Dans une deuxième partie, nous donnons quelques arguments en faveur d'une étude plus approfondie de ce mouvement. 


\title{
L’idéalisme britannique: histoire et actualité
}

\author{
SÉBASTIEN GANDON \\ Université Blaise Pascal, Clermont-Ferrand
}

MATHIEU MARION

Université du Québec à Montréal

\begin{abstract}
RÉSUMÉ. - L'idéalisme britannique est un mouvement qui a dominé les universités britanniques (et celles de son empire) pendant une cinquantaine d'années à la fin $d u x x^{e}$ siècle et au début du $x{ }^{e}$ siècle, mais qui est passé presque totalement inaperçu dans le monde francophone. Rejetés en bloc par les philosophes analytiques, ces auteurs ont aussi été ignorés pendant longtemps dans leur pays, mais certains d'entre eux, notamment Bradley et Collingwood, jouissent d'un regain d'intérêt à la faveur d'un renouveau des études sur les origines de la philosophie analytique. Ce texte est une introduction à l'idéalisme britannique, qui retrace, dans une première partie plus historique, les grandes lignes de sa genèse, son développement et son déclin. Dans une deuxième partie, nous donnons quelques arguments en faveur d'une étude plus approfondie de ce mouvement.
\end{abstract}

\begin{abstract}
British Idealism is a philosophical movement that dominated British universities (and those of its empire), for fifty years around the turn from the $X I X^{\text {th }}$ to the $X X^{\text {th }}$ century, but it went largely unnoticed in the French-speaking world. Condemned by analytic philosophers, these authors were also ignored in their own country, but some of them, notably Bradley and Collingwood, are now enjoying a newly found popularity within the larger trend towards a study of the origins of analytic philosophy. This text is an introduction to British Idealism that plots, in an historical first part, the outlines of its rise, development and decline. In the second part, we provide reasons for further studies of this movement.
\end{abstract}

L'expression «idéalisme britannique » désigne un mouvement philosophique qui est apparu dans la deuxième moitié du dix-neuvième siècle en GrandeBretagne. Les fréquentes références à Hegel dans les écrits de ses représentants expliquent aussi l'usage, pour décrire ce même mouvement, de l'expression «néo-hégélianisme », quoique la plupart de ces «idéalistes » ne donnèrent pas dans l'orthodoxie hégélienne ${ }^{1}$. Ce mouvement a connu son apogée dans

1. Voir H. Haldar, Neo-Hegelianism, Londres, Heath Cranton, 1927. Mais l'expression «néo-hégélianisme » a surtout été utilisée par les adversaires du mouvement dans un but polémique, comme le remarqua avec justesse R. G. Collingwood (Autobiography, Oxford: Clarendon Press, p. 15), pour en dénigrer les origines étrangères, en particulier à partir de la Première Guerre mondiale. Cet usage péjoratif est resté depuis, par exemple dans G. J. Warnock, English Philosophy since 1900, Londres, Oxford University Press, 1958, chapitre 1, ou, plus récemment, dans A. Quinton, "Absolute Idealism », Proceedings of the British Academy, vol. 57, 1971, 1-29. Pour un survol de la réception de Hegel en Grande Bretagne, voir J. Bradley, «Hegel in Britain: A Brief History of British Commentary and Attitudes », Heythrop Journal, vol. 20 (1979), p. 1-24 et 163-182. Il importe de bien comprendre que les idéalistes britanniques ne furent pas des 


\section{4· Philosophiques / Printemps 2009}

les universités britanniques au début du vingtième siècle, pour ensuite entrer dans une longue période de déclin, jusqu'à sa disparition quasi-totale dans les années quarante ${ }^{2}$, et il n'est aujourd'hui plus que l'objet de railleries, qui ont pour effet qu'on ne lit plus ces «idéalistes ${ }^{3}$. Même si tout choix de dates a un côté arbitraire, la parution de The Secret of Hegel de l'Écossais James Hutchison Stirling, en 1865, peut être considérée comme son point de départ ${ }^{4}$, tandis que la mort des grandes figures que furent Bernard Bosanquet, Francis Herbert Bradley, et John McTaggart Ellis McTaggart, entre 1923 et 1925, marque le début de sa fin. L'apogée du mouvement coïncide avec l'émergence d'une communauté philosophique à l'échelle nationale dans les pays de langue anglaise que sont l'Australie, le Canada et les États-Unis, et il n'est donc pas surprenant de voir l'idéalisme britannique jouer un rôle clef dans ce contexte ${ }^{5}$. Il faut ajouter à cette liste l'Afrique du Sud et le Raj, c'est-à-dire le sous-

«hégéliens » dans un sens orthodoxe; ils ont assimilé l'idéalisme allemand dans un contexte philosophique qui était le leur, leur pensée était tout à fait originale, et ils ne firent que des emprunts, aussi importants fussent-ils dans certains cas, à Hegel. Comme le dit bien James Bradley: «Hegel ne fut à peine plus qu'une ressource» (ibid., p. 14). (Pour l'opinion contraire, voir A. Quinton, "Absolute Idealism », op. cit., p. 18sq.) Certes, certains empruntèrent un concept d'absolu, considéré comme un être spirituel pour T. H. Green, comme la totalité de l'expérience immédiate pour F. H. Bradley, ou comme une communauté d'âmes immortelles mutuellement en amour pour J. M. E. McTaggart. Mais presqu'aucun d'entre eux ne reprit sa dialectique, et la plupart refusèrent l'identification de la pensée à la réalité, qui est au centre de la philosophie d'Hegel. Voir, sur ce dernier point et pour le cas de F. H. Bradley, son rejet sans appel dans The Principles of Logic, Londres, Oxford University Press, $2^{\mathrm{e}}$ éd., 1922, vol. II, p. 591. Sur cette question chez Bradley, voir J. W. Allard, The Logical Foundations of Bradley's Metaphysics: Judgment, Inference, and Truth, Cambridge, Cambridge University Press, 2005, chap. 1; J. Bradley, «F. H. Bradley's Metaphysics of Feeling and its Place in the History of Philosophy ", dans A. Manser \& G. Stock (dir.), The Philosophy of F. H. Bradley, Oxford, Clarendon Press, 1984, p. 227-242.

2. Certes, l'idéalisme n'a pas tout à fait disparu à ce moment-là, il reste des figures mineures comme T. M. Knox et G. R. G. Mure, influencées par l'enseignement de Collingwood, mais il reste très largement minoritaire et ne se renouvelle plus. Certains «idéalistes » de premier plan ont vécu très longtemps, comme l'Américain Brand Blanshard et l'Anglais Michael Oakeshott, qui sont morts, respectivement, en 1987 et en 1990. De nos jours, le Sud-Africain Errol E. Harris, devenu centenaire, est probablement le dernier survivant de cette époque. Un courant d'études de l'idéalisme britannique a cependant vu le jour depuis chez des auteurs dont l'œuvre est restée fortement influencée par l'idéalisme britannique, comme, par exemple, au Canada, Leslie Armour, James Bradley et William Sweet. Voir W. Sweet (dir.), Idealism, Metaphysics and Community: A Festschrift for Leslie Armour, Aldershot, Ashgate, 2001.

3. Certaines de ces railleries sont discutées dans la contribution de Bill Mander à ce numéro, "Les idéalistes britanniques et la poésie".

4. J. H. Stirling, The Secret of Hegel: Being the Hegelian System in Origin, Principle, Form and Matter, Londres, Longman, Roberts \& Green, 1865.

5. Dans le cas de l'Australie et du Canada, cette influence s'effectue par le biais de l'immigration de philosophes britanniques, tel John Watson, un étudiant de Caird à Oxford qui fit carrière au Canada. Voir les études de S. A. Grave, A History of Philosophy in Australia, St. Lucia, University of Queensland Press, 1984, en particulier le chapitre 2, et L. Armour \& E. Trott, The Faces of Reason. An Essay on Philosophy and Culture in English Canada 1850-1950, Waterloo, Wilfrid Laurier University Press, 1981. Sur John Watson, voir H. Haldar, Neo Hegelianism 
continent indien sous domination britannique ${ }^{6}$. Ce mouvement a donc «dominé» l'ensemble de la philosophie de langue anglaise pendant plus d'un demisiècle, mais, contrairement à d'autres mouvements de la même époque et d'une ampleur tout aussi significative, tels le néo-kantisme en Allemagne ou l'école de Brentano dans l'empire austro-hongrois, il est passé - et reste encore - presque totalement inaperçu en France. (Il faut dire qu'il en va de même de nos jours avec les grandes figures du kantisme français de la période, en France comme à l'étranger: qui se soucie encore de Lachelier, de Lagneau ou de Renouvier ?) Jean Wahl fut, entre les deux guerres, un des rares Français à s'intéresser à la philosophie de langue anglaise, avec deux études récemment rééditées: Les philosophies pluralistes d'Angleterre et d'Amérique (1920) et Vers le concret $(1932)^{7}$. Mais Wahl s'intéresse déjà aux adversaires de l'idéalisme, tels que William James et A. N. Whitehead, et se tourne rapidement vers l'existentialisme, de Kierkegaard à Gabriel Marcel - notons que ce dernier publiera en 1945 un recueil d'études (publiées dès 1918-1919) sur l'idéaliste américain Josiah Royce ${ }^{8}$. Après la guerre, Wahl formera quelques étudiants ${ }^{9}$ et organisera, au début des années soixante, le Colloque de Royaumont, qui fit découvrir la philosophie analytique en France ${ }^{10}$. Les quelques études publiées dans les années cinquante et soixante sur l'idéalisme

op. cit., chapitre 9 et L. Armour et E. Trott, The Faces of Reason, op. cit., chapitres 7 et 8 . Le cas des États-Unis est différent, car il y avait déjà un intérêt marqué pour Hegel dans le «transcendantalisme » (doctrine qui laissa des traces en littérature chez Emerson et Thoreau, et pour laquelle Madame de Staël, Coleridge et Carlyle sont aussi des sources), et dans les clubs philosophiques de St. Louis, de Concord et de Boston. Notons toutefois que le pragmatisme de Charles Sanders Peirce, malgré l'importance de Hegel, trouve aussi ses sources chez Reid et Hamilton. Voir sur ces questions les études contenues dans M. H. Fisch, Peirce, Semeiotic and Pragmatism, Bloomington IN, Indiana University Press, 1986, en particulier les chapitres 8 et 14, et R. A. Smyth, Reading Peirce Reading, Lanham MA, Rowman \& Littlefield, 1997, chapitre 1. L'influence de l'idéalisme britannique se fera surtout sentir chez Royce et, de manière négative, chez William James et John Dewey, ces derniers critiquant explicitement le monisme absolu de Bradley.

6. Ainsi, pour ne prendre qu'un exemple, Muhammad Iqbal, poète national du Pakistan, a aussi produit une œuvre philosophique importante, où la pensée de son professeur à Cambridge, J. M. E. McTaggart, joue un rôle de premier plan.

7. J. Wahl, Les philosophies pluralistes d'Angleterre et d'Amérique, Paris, Les empêcheurs de tourner en rond/Le Seuil, 2005 ; Vers le concret, Paris, Vrin, 2004. Il ne faut pas oublier une très rare étude, publiée deux ans après la mort de Bradley: E. Duprat, "La métaphysique de Bradley", Revue philosophique, vol. 52 (1926), p. 31-69.

8. G. Marcel, La métaphysique de Royce, Paris, Montaigne, 1945. De Gabriel Marcel, signalons en outre Coleridge et Schelling, Paris, Aubier-Montaigne, 1971.

9. Citons notamment François Houang, qui publia deux études sur la philosophie de Bosanquet: F. Houang, Le néo-hégélianisme en Angleterre. La philosophie de Bernard Bosanquet 1848-1923, Paris, Vrin, 1954; De l'humanisme à l'absolutisme: l'évolution de la pensée religieuse du néo-hégélien anglais Bernard Bosanquet, Paris, Vrin, 1954.

10. Cahiers de Royaumont, La philosophie analytique, Paris, Éditions de Minuit, 1962. Bien sûr, l'effet de cette découverte a été pendant de longues décennies essentiellement négatif. 


\section{6· Philosophiques / Printemps 2009}

britannique n'ont guère attiré l'attention ${ }^{11}$, et l'intérêt pour ce mouvement n'a pas survécu au dédain, généralisé à cette époque, pour la culture de langue anglaise. La philosophie analytique, que Wahl ne prisait surtout pas, a acquis depuis un droit de cité dans la sphère philosophique francophone, mais ses sources idéalistes restent encore très mal connues.

À l'exception des textes de Giuseppina D'Oro et de W. J. Mander, les contributions à ce numéro spécial de la revue Philosophiques ont leur origine dans un colloque international intitulé «Comment être idéaliste? Bradley et Collingwood» qui a eu lieu en juin 2006 à l'Université Blaise Pascal de Clermont-Ferrand, à notre connaissance le premier à se tenir en France sur ce sujet. En guise d'introduction, nous brosserons dans ce qui suit un rapide portrait historique de cet "idéalisme britannique ${ }^{12}$, pour que le lecteur puisse y trouver son chemin et disposer de quelques indications bibliographiques. Nous insisterons sur la dimension politique de ce mouvement, qui n'est pas abordée dans les contributions à ce numéro. Nous présenterons ensuite quelques-unes des raisons pour lesquelles, ici comme ailleurs, l'ignorance n'est pas une vertu.

11. Aux études de Houang, citées dans la note 9, il faut ajouter celles-ci (citées par ordre chronologique): J. Pucelle, L'idéalisme en Angleterre de Coleridge à Bradley, Neuchâtel, Éditions de la Baconnière, 1955 ; J. Pucelle La nature et l'esprit dans la philosophie de T. H. Green. La renaissance de l'idéalisme en Angleterre au XIX ${ }^{e}$ siècle, Louvain, Nauwelaerts, 1960 ; J. de Marneffe, La preuve de l'absolu chez Bradley, Paris, Beauchesne, 1961; C. Le Chevalier, Éthique et idéalisme: le courant néo-hégélien en Angleterre. Bernard Bosanquet et ses amis, Paris, Vrin, 1963; P. Fruchon, Francis Herbert Bradley: les présupposés de l'histoire critique. Étude et traduction, Paris, Les Belles Lettres, 1965. Il n'existe que peu d'études plus récentes, signalons: J. Bradley, "Relations, intelligibilité et non-contradiction dans la métaphysique du sentir de F. H. Bradley: une réinterprétation ", Archives de philosophie, vol. 54, 1991, p. 529-551 et vol. 55, 1992, p. 77-91 ; J.-P. Narboux, "Négation, contrariété et contradiction : sur la théorie éliminativiste de la négation dans l'idéalisme anglais ", Archives de philosophie, vol. 68, 2005, p. 419446; M. Girel, "Relations internes et relations spatiales: James, Bradley et Green ", Archives de philosophie, vol. 69, 2006, p. 395-414. (Voir aussi l'introduction de ce dernier à J. Wahl, Vers le concret, op. cit.)

12. Le lecteur peut consulter, outre l'étude de Jean Pucelle, L'idéalisme en Angleterre de Coleridge à Bradley, op. cit., les études d'ensemble suivantes, qui datent cependant d'avant la Seconde Guerre mondiale: A. K. Rogers, English and American Philosophy since 1800. A Critical Survey, New York, MacMillan, 1922; H. Haldar, Neo-Hegelianism, op. cit.; W. R. Sorley, A History of English Philosophy, Cambridge, Cambridge University Press, 1937; R. Metz, A Hundred Years of British Philosophy, Londres, Allen \& Unwin, 1938. Il faut aussi signaler deux études de la même époque, plus systématiques et moins historiques: C. R. Morris, Idealistic Logic. A Study of its Aim, Method and Achievement, Londres, MacMillan, 1933 ; A. C. Ewing, Idealism: A Critical Survey, Londres, Methuen, 1934. Après la guerre, on ne trouve que des études partielles, comme les chapitres 3 et 4 de J. Passmore, A Hundred Years of Philosophy, $2^{\mathrm{e}}$ éd., Harmondsworth, Penguin Books, 1966. Le chapitre sur l'idéalisme dans l'étude de John Skorupski, English-Language Philosophy 1750-1945 (Oxford, Oxford University Press, 1993) est certes plus récent et fort lucide, mais son étude est thématique et son auteur n'a pas cherché à être exhaustif. 


\section{1. «The Rhine has flowed into the Thames »}

Metaphysics is the finding of bad reasons for what we believe upon instinct.

F. H. BRADLEY

La domination presque sans partage de la philosophie analytique en GrandeBretagne à partir de la fin des années quarante a relégué l'idéalisme britannique au rang de curiosité historique. Cette domination a contribué à forger une image faussement monolithique de la pensée philosophique de langue anglaise, en laissant entendre que l'idéalisme ne serait qu'un égarement, une aberration temporaire causée par l'influence d'une philosophie d'origine continentale (l'idéalisme allemand, de Kant à Hegel et Lotze ${ }^{13}$ ), tandis qu'il existerait une continuité de nature entre l'empirisme des dix-huitième et dix-neuvième siècles (en gros, de Hobbes à Mill) et la philosophie analytique au vingtième siècle. Cette vision est fausse. Non seulement l'idée selon laquelle la philosophie analytique n'est qu'une continuation de l'empirisme donne une image réductrice de celle-ci (de surcroît, elle nie ce que la philosophie analytique doit, ne serait-ce que par réaction, à l'idéalisme britannique), mais, plus encore, l'idée selon laquelle le mouvement idéaliste aurait été un égarement (de plus d'un demi-siècle!) est le corrélat d'une méconnaissance profonde de l'histoire de la pensée britannique (c'est-à-dire anglaise et écossaise), autrement plus riche en spéculations métaphysiques que la lecture de Hume et de Ayer pourrait le laisser croire $^{14}$.

En effet, l'étude de John H. Muirhead, The Platonic Tradition in AngloSaxon Philosophy ${ }^{15}$, a montré qu'il existe à l'intérieur de la pensée anglaise une tradition qui ne présuppose pas les thèses empiristes, dont l'origine se trouve chez Jean Scot Érigène et que l'on retrouve, au dix-septième siècle, chez les «platoniciens de Cambridge» (Henry More, Ralph Cudworth, et al.). Cette

13. Sur l'importance qu'a eue Lotze dans l'idéalisme britannique, voir plus loin.

14. La critique de la métaphysique dans le Cercle de Vienne, adoptée entre autres par A. J. Ayer dans Language, Truth and Logic ( $2^{\mathrm{e}}$ éd., Londres, Victor Gollancz, 1946), a longtemps servi de repoussoir contre la philosophie analytique (cette généralisation est simplement fausse, puisqu'en réalité les philosophes analytiques ont beaucoup contribué à la métaphysique, à commencer par Russell). Ayer, qui approuvait le mot de Hume condamnant les ouvrages de métaphysique aux flammes (Language. Truth and Logic, op. cit., p. 54), citait par ailleurs « une phrase prise au hasard " dans un livre de Bradley comme exemple de non-sens métaphysique (ibidem, p. 6). Ce genre de propos a fait un grand tort à la réputation de Bradley.

15. J. H. Muirhead, The Platonic Tradition in Anglo-Saxon Philosophy, Londres, Allen \& Unwin, 1931. Voir aussi, J. H. Muirhead, "Past and Present in Contemporary Philosophy ", dans J. H. Muirhead (dir.), Contemporary British Philosophy. First Series, Londres, Allen \& Unwin, 1924, p. 309-324. Nous ne voudrions pas laisser croire que les thèses de Muirhead sont le dernier mot - voir, par exemple, les critiques dans J. Bradley, "Hegel in Britain... ", op. cit., p. 2-12 -, elles servent ici simplement à illustrer la richesse de la philosophie britannique, trop souvent présentée de façon caricaturale et réductrice comme "empiriste ", pour être ensuite rejetée du revers de la main. 
tradition aurait ensuite subi une éclipse au siècle suivant, où on ne retrouve que les épigones oxoniens de Malebranche, Arthur Collier et John Norris, et, bien sûr, George Berkeley. Un intérêt pour l'idéalisme allemand s'est ensuite manifesté dès le début du dix-neuvième siècle dans les milieux littéraires anglais, grâce à l'immense influence du poète Samuel Taylor Coleridge qui discute Kant, Fichte et Schelling dans Biographia Literaria (1817) et Aids to Reflection $(1825)^{16}$ — et, par la suite, grâce à Thomas Carlyle, auteur de Sartor Resartus (1833-34), On Heroes, Heroworship and the Heroic in History (1841) et de nombreux essais sur la philosophie allemande (la pensée de Carlyle influencera à son tour des figures aussi diverses qu'Emerson, Green, Nietzsche et Ruskin). C'est aussi l'époque où l'auteur des Confessions d'un mangeur d'opium anglais (1822), Thomas de Quincey, publie Les derniers jours d'Emmanuel Kant (1827) et Thomas Love Peacocke, de savoureuses satires philosophiques.

Cependant, le milieu philosophique était durant cette période dominé par deux grandes écoles, celle de la tradition empiriste de Locke, Berkeley et Hume, et celle de l'école écossaise du «common sense ", inspirée de Thomas Reid. L'empirisme, allié à la fois à l'associationnisme en psychologie (dont il est en fait l'origine) et à l'utilitarisme de Bentham, était développé par John Stuart Mill et trouvait son expression politique dans le «radicalisme» dont Élie Halévy nous a légué une admirable étude ${ }^{17}$, tandis que la philosophie de Reid était poursuivie, entre autres, par Thomas Brown, Dugald Stewart et William Hamilton - auteurs que l'on traduisit rapidement en France, sous l'impulsion de Victor Cousin, mais qui furent ensuite relégués aux oubliettes. Peu de ces philosophes connaissaient l'allemand, qui n'avait pas à l'époque le statut de langue philosophique qu'on lui attribue de nos jours, et leur connaissance d'un Hegel était indirecte, essentiellement, à cause des liens culturels privilégiés de l'époque entre la France et l'Écosse, à travers De l'Allemagne de Madame de Staël (1814) et, surtout, les écrits de Cousin. C'est dans ce contexte que deux jeunes Écossais lisant Hegel dans le texte, James Frederick Ferrier et James Hutchison Stirling, jetèrent les bases du mouvement idéaliste avec, respectivement, The Institutes of Metaphysic en 1854 (une présentation more geometrico unique depuis Spinoza) et The Secret of Hegel en $1865^{18}$. C'est à Oxford, cependant, que le mouvement connut un véritable essor grâce à l'enseignement au collège Balliol de Benjamin Jowett, traducteur de Platon, qui introduisit l'étude de Hegel et, surtout, grâce à ceux d'Edward Caird et

16. Voir J. H. Muirhead, Coleridge as Philosopher, Londres, Allen \& Unwin, 1930, ainsi que R. Wellek, Immanuel Kant in England 1793-1838, Princeton, Princeton University Press, 1931.

17. E. Halévy, La formation du radicalisme philosophique, 3 vol., Paris, P.U.F., 1995.

18. J. F. Ferrier, The Institutes of Metaphysic: The Theory of Knowing and Being, Edimbourg, W. Blackwood, 1854; et J. H. Stirling, The Secret of Hegel, op. cit. 
de Thomas Hill Green ${ }^{19}$. Malgré une mort précoce en 1882, Green allait changer le cours de la philosophie dans cette université. Pour lui, il fallait «fermer Mill et Spencer, pour ouvrir Kant et $\mathrm{Hegel}^{20}{ }^{2}$. Green exerça une influence profonde sur ses étudiants, et c'est avec lui que l'idéalisme revint sur le devant de la scène philosophique britannique en cette seconde partie du dix-neuvième siècle. Cette tradition idéaliste, qui court de More et Cudworth à Green et Bradley, en passant par Coleridge et Carlyle, constitue donc une des facettes marquantes d'une pensée britannique plus riche qu'on ne le pense d'ordinaire, au même titre que la tradition empiriste, la philosophie du «common sense ", et d'autres développements à l'intérieur du courant matérialiste et naturaliste, auxquels appartiennent, entre autres, William Whewell, Charles Darwin, Thomas Huxley, Herbert Spencer, W. S. Jevons, W. K. Clifford et Leslie Stephen (père de Virginia Woolf), et auxquels il faudrait ajouter les positivistes influencés par Comte, tels que George Henry Lewes (dont George Eliot fut la maîtresse). Ce courant est resté vivace dans la société britannique au cours du dix-neuvième siècle, mais surtout chez les scientifiques et hors des milieux académiques; il n'aura que peu d'influence dans les départements de philosophie.

La référence à Coleridge et celle, constante, aux poètes romantiques anglais, de Wordsworth à Tennyson et Browning, qui fait l'objet de l'article de Bill Manders dans ce volume ${ }^{21}$, mérite d'être soulignée. Il faut éviter de ranger, comme le font Muirhead et Pucelle ${ }^{22}$, Coleridge et les idéalistes britanniques en une seule tradition, car ces derniers ne retinrent à peu près rien des vues philosophiques de Coleridge. Au contraire, ils croyaient pouvoir fournir sur une autre base une explication philosophique des intuitions métaphysiques de ces poètes ${ }^{23}$.

L'année de la parution de Secret of Hegel, $1865^{24}$, peut servir de ligne de partage des eaux, car c'est aussi celle de la publication de An Examination

19. Pour Muirhead, le mouvement idéaliste doit autant au retour à Platon initié par Jowett, qu'à la découverte de Hegel. Voir J. H. Muirhead, The Platonic Tradition in AngloSaxon Philosophy, p. 413.

20. F. Houang, Le néo-hégélianisme en Angleterre, op. cit., p. 15. Pour un commentaire de cet ordre, voir la toute dernière phrase de sa très longue critique de l'empirisme dans "Introductions to Hume's 'Treatise of Human Nature' " dans Works of Thomas Hill Green, R. L. Nettleship (dir.), Londres, Longmans, Green \& Co., 3 vol., 1885-1888, vol. 1, p. 371.

21. Voir aussi la très intéressante note de James Bradley, «Hegel in Britain: A Brief History of British Commentary and Attitudes ", op. cit., p. 17, note 39.

22. J. H. Muirhead, The Platonic Tradition in Anglo-Saxon Philosophy, op. cit.; J. Pucelle, L'idéalisme en Angleterre, op. cit.

23. Voir, par exemple, les premières pages de T. H. Green, Prolegomena to Ethics, Oxford, Clarendon Press, $5^{\mathrm{e}}$ éd., 1906, p. 1-3 et, pour la conception que se faisaient les idéalistes des rapports entre la philosophie et la poésie, voir l'article de Bill Mander dans ce volume,

24. On mesure l'écart entre les deux traditions philosophiques lorsqu'on réalise que c'est en 1865 qu'Otto Liebmann prononça son fameux mot d'ordre du « retour à Kant », alors que les Britanniques découvraient à peine Hegel. 
of Sir William Hamilton's Philosophy, de John Stuart Mill ${ }^{25}$. Avec cet ouvrage volumineux, Mill donna un coup d'arrêt à l'école écossaise du «common sense» de Reid, dont Hamilton fut donc l'ultime grand représentant. Mill avait beau jeu d'étaler les contradictions internes d'un syncrétisme cherchant à allier le «common sense» de Reid à la philosophie kantienne. Son ouvrage allait créer beaucoup de remous, s'attirant de nombreuses répliques, dont celle, l'année suivante, du Waynflete Professor of Metaphysics à Oxford, Henry Longueville Mansel, dans The Philosophy of the Conditioned ${ }^{26}$. Mansel était aussi opposé à l'influence grandissante de l'idéalisme, mais il quitta Oxford en 1867, laissant le champ libre à l'influence de Green. Ce dernier était en poste depuis 1860 et fut nommé Whyte's Professor of Moral Philosophy en 1877. La période fut celle de l'ascendance de Green sur les nouvelles générations à Oxford: parmi ses étudiants à la fin des années soixante, on trouve F. H. Bradley (1865-69), Bernard Bosanquet (1867-70) et John Cook Wilson $(1868-73)^{27}$. Un autre étudiant, William Wallace, qui se distinguera surtout par ses traductions de Hegel, deviendra son successeur (1882-1897), tandis que Caird, parti rejoindre son frère John à Glasgow (qui fut un autre foyer, tout aussi important, du mouvement idéaliste), reviendra à Oxford en 1893 pour diriger le collège Balliol.

Les causes de l'engouement pour l'idéalisme sont nombreuses. Au premier chef, il faut noter la transformation de la société britannique sous l'effet de la révolution industrielle, dont les conséquences désastreuses sur la classe ouvrière comme sur l'environnement étaient patents. Ce bouleversement fut associé un peu rapidement à l'hédonisme utilitariste, dont Green va fournir une importante critique. Par ailleurs, à Oxford, à l'époque de Green, la plupart des étudiants en philosophie provenaient du presbytère et se destinaient à y retourner, et une philosophie religieuse comme celle des

25. J. S. Mill, An Examination of Sir William Hamilton's Philosophy, Toronto/Londres, University of Toronto Press/Routledge \& Kegan Paul, 1979.

26. H. L. Mansel, The Philosophy of the Conditioned, Londres, Alexander Strahan, 1866.

27. Cook Wilson contribuera cependant à la disparition de l'idéalisme par la suite, en devenant le père de l'école «réaliste» d'Oxford — qui s'opposait tout autant à l'empirisme dont la continuation directe fut la «philosophie du langage ordinaire» de J. L. Austin. Sur Cook Wilson, voir M. Marion, "Cook Wilson, John", dans The Dictionary of TwentiethCentury British Philosophers, Bristol, Thoemmes Press, 2005, p. 187-192 et M. Marion, "Introduction", dans J. Cook Wilson, Statement and Inference, Bristol, Thoemmes Press, 2002, p. v-xxvii. Sur l'école réaliste d'Oxford, la philosophie du langage ordinaire, et leur opposition au phénoménisme d'inspiration empiriste, voir M. Marion, "Oxford Realism: Knowledge and Perception ", British Journal for the History of Philosophy, vol. 8, 2000, p. 299-338 et 485-519. Pour une relecture de Bradley à la lumière des remarques de Collingwood (dans «The Metaphysics of F. H. Bradley: An Essay on 'Appearance and Reality' ", publié récemment dans An Essay on Philosophical Method, $2^{e}$ éd., Oxford: Clarendon Press, p. 227-252) selon lesquelles Bradley et Cook Wilson étaient en fait alliés contre le phénoménalisme, voir G. Stock, "Introduction: The Realist Spirit in Bradley's Philosophy », dans G. Stock (dir.), Appearance and Reality, Oxford, Clarendon Press, 1998, p. 1-18. 
idéalistes, si «radicale» fût-elle ${ }^{28}$, était beaucoup plus attrayante que celle des utilitaristes et des "philosophical radicals", associés à travers leur athéisme aux Lumières et à la Révolution française. Cependant, Green se distingue aussi par son idéal d'émancipation: selon Caird, il possédait une sensibilité "démocratique ou chrétienne, qui ne pouvait pas tolérer l'idée de privilège, et désirait pour toute classe et tout individu une part entière dans le vaste héritage de l'humanité29 ". À l'encontre du "laissez-faire » de l'école de Manchester (Richard Cobden, John Bright), la «philosophie sociale» de Green avait pour résultat de légitimer l'intervention de l'État, lorsque celui-ci agit pour promouvoir la réalisation de soi des individus.

Par ailleurs, la philosophie de Green constituait pour certains un rempart contre les approches positivistes et athéistes de l'histoire biblique ${ }^{30}$, et contre la montée du naturalisme, en particulier, à travers l'évolutionnisme de Darwin, qui menaçaient le credo religieux. La parution de son grand ouvrage, On the Origins of Species quelques années auparavant, en $1859^{31}$, avait causé une énorme controverse, dont témoigne le célèbre débat entre Huxley et Wilberforce à Oxford, en 1860. L'évolutionnisme sera popularisé par l'agnostique Herbert Spencer, dont les livres se vendront à des centaines de milliers d'exemplaires et qui sera la cible de bien des attaques ${ }^{32}$. (Il ne faut cependant pas tirer la conclusion que les idéalistes britanniques rejetèrent en bloc les thèses de Darwin; au contraire, plusieurs d'entre eux croyaient pouvoir concilier, à travers leur réinterprétation de Hegel, leur credo religieux avec l'évolutionnisme ${ }^{33}$.)

28. Voir là-dessus la remarque de William James dans Pragmatism. A New Name for Some Old Ways of Thinking, New York, Longmans, Green \& Co., 1907, p. 17.

29. T. H. Green, Prolegomena to Ethics, op. cit., p. vii.

30. L'écrivain George Eliot a traduit à l'époque, et publié sous son vrai nom, Marian Evans, deux livres importants: D. F. Strauss, The Life of Jesus Critically Examined, Londres, Chapman, 1846, et L. Feuerbach, The Essence of Christianity, Londres, Chapman, 1854, qui sont autant d'exemples... d'hégélianisme de gauche.

31. C. Darwin, On the Origins of Species by means of Natural Selection or the Preservation of Favourite Races in the Struggle for Life, Londres, John Murray, 1859; traduction française: L'origine des espèces au moyen de la sélection naturelle ou la lutte pour l'existence dans la nature, Paris, C. Reinwald et Cie., 1876.

32. Voir entre autres ses Principles of Biology, 2 vol., Londres, Williams \& Norgate, 1864. Pour une étude récente, voir M. Francis, Herbert Spencer and the Invention of Modern Life, Ithaca NY., Cornell University Press, 2007.

33. Voir, par exemple, l'article «Darwin and Hegel» dans D. G. Ritchie, Darwin and Hegel with other Philosophical Studies, Londres, Swan Sonnenschein, 1893, 38-76. Pour William Wallace, «l'axiome fondamental» de Hegel selon lequel le réel est rationnel n'est... «après tout qu'une autre manière d'affirmer la survie du mieux adapté (maintenance of the fittest) " (Prolegomena to the Study of Hegel's Philosophy, $2^{\mathrm{e}}$ éd., Oxford, Clarendon Press, 1894, p. 61-62). L'idée peut paraître absurde, mais il faut noter que Spencer, qui avait proposé une forme d'évolutionnisme avant Darwin, avait repris une idée de Schelling relayée par von Bader. 
Dans «Popular Philosophy in its Relation to Life » ${ }^{34}$, Green dresse le portrait des développements en éthique depuis le siècle des Lumières, et il y constate une "erreur métaphysique ", celle d'avoir voulu abstraire l'individu de la société, pour l'animaliser ${ }^{35}$. Tous ces développements partent du «sentir» (feeling), et conçoivent l'individu en termes d' «appétits» et d'une faculté lui permettant de calculer le meilleur moyen de réaliser ses désirs - l'équilibre social étant conçu en termes d'ajustement des comportements de chacun. Green rejette tout cela pour mettre au centre de sa philosophie l'idée d'une réalisation spirituelle de soi, censée rendre compte du sentiment chrétien du "péché » ${ }^{36}$, et qui passe par une reconnaissance du caractère foncièrement social de l'individu, ce "moi commun" (en français dans le texte ${ }^{37}$ ). Dans ses Prolegomena to Ethics, publiés après sa mort en $1883^{38}$, il s'oppose donc à l'empirisme et au darwinisme en réclamant un retour à $\mathrm{Kant}^{39}$ : selon lui, ces deux doctrines conduisent à faire de la conscience un être qui émerge de la nature, ce qui ne serait qu'un bysteron proteron ${ }^{40}$. Tout en rejetant la chose-en-soi, Green récupère le moi transcendantal de Kant: la conscience est pour lui responsable de la mise en ordre des phénomènes, chose qu'elle ne peut accomplir que si elle est conçue comme étant en partie en dehors du temps et éternelle. Ce moi ne peut donc être que spirituel, ce qui est, toujours selon Green, la vérité contenue dans le mot de Kant «l'entendement crée la nature ${ }^{41}$ ». Il est aussi et surtout une reproduction d'un être suprême présupposé par l'existence du monde ${ }^{42}$, en d'autres termes plus directement chrétiens: Dieu crée l'homme à son image ${ }^{43}$. Si Dieu se reproduit dans l'âme humaine et que celle-ci en est consciente, alors elle est consciente qu'elle est perfectible, malgré ses limitations animales; elle doit donc chercher à réaliser ses propres virtualités, ce qu'elle ne peut faire qu'à travers la sociétét4. (On peut donc parler d'une forme de "perfectionnisme» moral chez Green ${ }^{45}$.) Ce n'est en effet que dans la mesure où nous sommes membres d'une société dont nous pouvons concevoir le bien commun que nous pouvons agir moralement, et «chacun doit d'abord remplir les devoirs qu'exige sa

34. Voir T. H. Green «Popular Philosophy in its Relation to Life», dans Works of Thomas Hill Green, op. cit., vol. III, p. 93-125.

35. Ibidem, p. 116-117.

36. Ibidem, p. 121.

37. Ibidem, p. 123. La référence est à Rousseau.

38. Mais aussi dès 1874 dans son introduction à l'édition du Treatise on Human Nature de Hume.

39. T. H. Green, Prolegomena to Ethics, op. cit., $\mathbb{} 8$.

40. Ibidem, $\mathbb{} 9$.

41. Ibidem, $\mathbb{S} 11$ et 38 .

42. Voir Works of Thomas Hill Green, op. cit., vol. III, p. 145.

43. Ibidem, $\mathbb{S} 99$. Voir aussi T. H. Green, Works, op. cit. vol. III, p. 227.

44. Ibidem, $\mathbb{S}$ 190-191.

45. Parmi les nombreuses études sur la philosophie morale de Green, signalons G. Thomas, The Moral Philosophy of Thomas Hill Green, Oxford, Clarendon Press, 1987; B. Wempe, T. H. Green's Theory of Positive Freedom, Exeter, Imprint Academic, 2004. 
place dans la sociétét ${ }^{46}$. Pour Green, un citoyen est donc autonome et rationnel en ce qu'il perçoit ce bien commun et qu'il internalise les normes communes — chrétiennes - afin de guider ses actions. Partis de Kant, nous nous sommes rapprochés de "l'état protestant" de Hegel ou encore de l'idéal de société, protestante mais laïcisée, que l'on retrouve chez Lotze ${ }^{47}$. Par ailleurs, Green reste un libéral dans la lignée de Mill, puisqu'il considère que l'État ne doit agir que pour éliminer les obstacles à cet agir moral ${ }^{48}$.

Cette "philosophie sociale» fut très populaire auprès d'étudiants en philosophie qui étaient en majorité destinés, à Oxford, au presbytère protestant - elle sera aussi perçue comme menant à une forme de libéralisme autre que celui des radicaux, voire vers une forme de socialisme chrétien ${ }^{49}$, et débordera le cadre de l'université pour influencer l'ensemble de la Grande-Bretagne, dans les dernières décennies de l'ère victorienne. En effet, par son influence sur des figures comme les réformistes sociaux Arnold Toynbee (à ne pas confondre avec l'historien du même nom, qui est son fils) et Alfred Milner ${ }^{50}$, sur des personnalités religieuses comme l'évêque socialiste Charles Gore, sur des politiciens comme Henry Asquith (Premier ministre libéral de 1908 à 1916) et R. B. Haldane (deux fois nommé Lord Chancellor) ${ }^{51}$, et à travers celle de

46. Ibidem, $\$ 183$.

47. Voir là-dessus l'étude de Philippe Devaux, Lotze et son influence sur la philosophie anglo-saxonne. Contribution à l'étude historique et critique de la notion de valeur, Bruxelles, Lamertin, 1932, p. 25. La traduction anglaise du Mikrokosmus de Lotze, où l'on retrouve l'exposé de ses vues sur la société, a connu une grande popularité, probablement parce qu'il a aussi cherché à concilier la science avec un respect des valeurs morales et religieuses du protestantisme: Microcosmus. An Essay Concerning Man and his Relation to the World, 2 vol., Edimbourg, T. \& T. Clark, 1885. Pour une étude de la tentative de réconciliation, voir G. Santanaya, «Lotze's Moral Idealism ", Mind, vol. 15 (1890), p. 191-212.

48. T. H. Green, Lectures on the Principles of Political Obligation, Londres, Longmans, Green and Co., 1911, p. 208-209. Bien sûr, il y des divergences entre le libéralisme de Mill et de Green: ce dernier peut, par exemple, défendre l'intervention de l'État dans le domaine privé par une loi sur la tempérance en faisant valoir que l'alcoolisme est un frein à la réalisation de soi pour les individus alcooliques; dans les termes mêmes du libéralisme de Mill, une telle loi n'est cependant pas admissible.

49. Selon Adam Ulam, Green est un des ancêtres du parti travailliste. Voir A. B. Ulam, Philosophical Foundations of English Socialism, Cambridge MA, Harvard University Press, 1951.

50. Milner s'est par ailleurs distingué en Afrique du Sud, où il fut gouverneur de la colonie du Cap durant la guerre des Boers, pour sa défense de l'impérialisme britannique, ce qui a entaché fortement le mouvement idéaliste. (Voir ci-dessous.) Dans les années trente, le lobby des "appeasers", favorables à la conciliation avec Hitler, était aussi formé de nombreuses figures dont le passé était lié à Lord Milner et à l'Afrique du Sud, quoique ce fut un idéaliste, R. G. Collingwood, qui les dénonça comme les héritiers du réalisme de Cook Wilson, dans son Autobiography, op. cit., p. 167.

51. Parmi les politiciens de l'époque, A. J. Balfour, qui fut premier ministre de 1902 à 1905 (et, plus tard, responsable de la "déclaration Balfour» sur la Palestine) était aussi un philosophe - ce qui montre à quel point peut être fausse l'affirmation qu'en Angleterre il n'y a pas eu d'«intellectuels» qui ont joué un rôle public —, mais il était un Tory et n'était pas un épigone de Green; voir A. J. Balfour, "Green's Metaphysics of Knowledge », Mind, vol. 9, 1884, p. 73-92, et «A Criticism of Current Idealistic Theories ", Mind, n.s., vol. 2, 1893, p. 425-440. 
philosophes politiques comme Henry Jones et David George Ritchie, la philosophie sociale de Green a joué un rôle fondamental dans l'histoire de la pensée libérale et dans l'émergence d'un socialisme chrétien en Grande-Bretagne à la fin de l'ère victorienne ${ }^{52}$. Green a également influencé Bradley, pour qui, dans "My Station and its Duties ", l'individu est un " universel concret ${ }^{53}$ " à l'intérieur de l'organisme social; conçu indépendamment de l'ensemble des relations qui constituent ce dernier, l'individu n'existe tout simplement $\mathrm{pas}^{54}$. Bradley ${ }^{55}$, et à un degré moindre Bosanquet ${ }^{56}$, furent cependant perçus comme des conservateurs.

52. Voir à ce sujet: A. J. M. Milne, The Social Philosophy of English Idealism, London, Allen \& Unwin, 1962; M. Richter, The Politics of Conscience: T. H. Green and his Age, Londres, Weidenfeld \& Nicholson, 1964; S. den Otter, British Idealism and Social Explanation, Oxford, Clarendon Press, 1996; M. Carter, T. H. Green and the Development of Ethical Socialism, Exeter, Imprint Academic, 2003; A. de Sanctis, The Puritan Democracy of Thomas Hill Green, Exeter, Imprint Academic, 2005. Parmi les nombreuses études sur l'idéalisme britannique et la philosophie politique, voir: D. Boucher et A. Vincent, British Idealism and Political Theory, Edimbourg, Ediburgh University Press, 2000; P. Nicholson, The Political Philosophy of the British Idealists, Cambridge, Cambridge University Press, 1990.

53. La notion d' « universel concret» (dont l'origine se trouve dans J. H. Stirling, The Secret of Hegel, op. cit., p. xxii, dont c'est le "secret ») a joué un rôle fondamental dans l'idéalisme britannique, mais elle est aujourd'hui mal comprise. Voir à ce sujet les pages de François Houang dans Le néo-hégélianisme en Angleterre, op. cit., p. 29-30; pour une discussion récente, jetant cependant un doute sur les sources hégéliennes de cette notion, voir R. Stern, "Hegel, British Idealism, and the Curious Case of the Concrete Universal ", British Journal for the History of Philosophy, vol. 15 (2007), p. 115-153. Cette notion est aussi discutée dans les contributions de Bill Mander et de Mathieu Marion à ce numéro.

54. F. H. Bradley, "My Station and its Duties », dans Ethical Studies, $2^{\mathrm{e}}$ éd., Oxford, Clarendon Press, 1927, p. 166. Bradley reconnaît cependant qu'une société quelconque peut faire montre d'imperfection (p. 203-204) et doit en conséquence être réformée d'un point de vue idéal. Bradley est alors forcé d'introduire un concept de «moralité idéale » (ideal morality), que l'individu doit chercher à réaliser, mais qu'il considère en fin de compte contradictoire (p. 233-234), puisqu'elle dépend du concept du mal qu'elle cherche justement à dépasser. La possibilité d'une réalisation de soi, laissée ouverte du côté de l'expérience religieuse à la fin du volume, serait plutôt, dans les termes d'Appearance and Reality, de l'ordre d'une dissolution du soi dans l'expérience absolue.

55. Les arguments de Bradley (et de Bosanquet à sa suite) pourraient laisser craindre une dissolution de l'individu et de ses droits au profit de la communauté et de l'État, et Peter Geach a pu dire qu'ils rappellent «l'adoration de l'État par les nazis» (H. A. Lewis (dir.), Peter Geach: Philosophical Encounters, Dordrecht, Kluwer, 1991, p. 3). Cette accusation est gratuite car Bradley, mort en 1924, ne s'est jamais «engagé » pour quelque cause que ce soit - il est resté isolé de toute vie publique, vivant comme un ermite dans le collège Merton à Oxford.

56. Malgré ses activités politiques dans l'aile radicale du parti libéral, on a accusé Bosanquet de ne pas faire assez de place aux droits de l'individu face à l'État dans B. Bosanquet, The Philosophical Theory of the State, Londres, MacMillan, 1899. On le considère aussi «conservateur " à cause de certaines prises de position, par exemple, sur la guerre des Boers, ou encore à cause de sa défense du rapport majoritaire de la Poor Law Commission; le rapport minoritaire fut à l'origine de la réforme de la Poor Law (dont la première version date de 1601), qui créera le Welfare State à la fin des années quarante. Voir B. Bosanquet, "The Majority Report of the Poor Law Commission", The Sociological Review, vol. 2 (1909), p. 109-126. Bosanquet a beaucoup travaillé pour la Charity Organisation Society à Londres, qui avait donné son appui au rapport majoritaire, car il y voyait le débouché de sa philosophie sociale. Il avait même quitté son emploi à Oxford pour joindre cette société. Voir S. den Otter, British Idealism 
Mais, il importe de noter que, quelle qu'ait été sa destinée politique, la philosophie de Green a, par son influence, éclipsé peu à peu dans le domaine moral et politique l'utilitarisme de Bentham et de Mill, dont Henry Sidgwick deviendra virtuellement le seul représentant universitaire à la fin du dix-neuvième siècle $^{57}$. Lorsque George Edward Moore et Bertrand Russell font leurs études à Cambridge au début de la dernière décennie du dix-neuvième siècle, "Old Sidg » est perçu comme dépassé, et ils se tournent vers l'idéaliste McTaggart.

On peut donc dire que, dans la seconde partie du dix-neuvième siècle, l'école écossaise de Reid et Hamilton disparaît rapidement, que l'empirisme ${ }^{58}$ et l'utilitarisme subissent le même sort un peu plus tard, tandis que l'idéalisme est alors en plein essor. À la fin de la Première Guerre mondiale, la situation a totalement changé. Même à son apogée, l'idéalisme n'avait pas dominé les universités britanniques sans partage. À Manchester, Robert Adamson s'était rangé du côté du "réalisme » à la fin de sa vie, et Samuel Alexander et George Dawes Hick allaient poursuivre dans cette direction ${ }^{59}$. À Cambridge, Moore et Russell renièrent l'idéalisme de McTaggart dès le tournant du siècle — c'est un chapitre bien connu de l'histoire de la philosophie britannique, puisque cette « révolte» est une des sources de la philosophie analytique. Leur influence fut déterminante sur la philosophie des générations suivantes à Cambridge, dont celles de F. P. Ramsey, de Ludwig Wittgenstein, de R. B. Braithwaite, etc. ${ }^{60}$. À

and Social Explanation, op. cit., p. 180-195. Sur l'histoire de cette société, voir R. Humphreys, Poor Relief and Charity, 1869-1945: the London Charity Organization Society, Londres, Palgrave, 2001.

57. Sur l'œuvre de Sidgwick et son contexte, voir J. B. Schneewind, Sidgwick's Ethics and Victorian Moral Philosophy, Oxford, Clarendon Press, 1977.

58. Après la retraite d'Alexander Bain et la mort de George Croom Robertson à Aberdeen, à la fin du dix-neuvième siècle, l'empirisme n'a plus de représentant majeur dans les universités britanniques.

59. R. Adamson, The Development of Modern Philosophy with other Lectures and Essays, 2 vol., Edimbourg, William Blackwood \& Sons, 1903; S. Alexander, "The Basis of Realism ", Proceedings of the British Academy, vol. 4, 1914, 1-36; et Space, Time \& Deity, Londres, MacMillan, 1920; G. Dawes Hicks, Critical Realism. Studies in the Philosophy of Mind and Nature, Londres, MacMillan, 1938. Sur Alexander, il faut signaler les études de Philippe Devaux, Le système d'Alexander. Exposé critique d'une théorie néo-réaliste du changement, Paris, Vrin, 1929, "L'esprit du néo-réalisme anglais ", Revue internationale de philosophie, vol. 1, 1939, 499-541. (Sur ce pionnier dans le domaine que fut Devaux, voir, de Paul Gochet, qui fut son étudiant, "Philippe Devaux, découvreur de la pensée anglo-saxonne ", Chromatikon, vol. II, 2006, 151-160.)

60. Cette influence débordera les milieux académiques, puisque le réalisme de la «valeur intrinsèque » de Moore, adopté un temps par Russell, et l'épistémologie de ce dernier eurent une grande influence sur le "Bloomsbury Group ", et, à travers celui-ci, sur la littérature (E. M. Forster et Virginia Woolf) et la critique d'art britannique (surtout Roger Fry) entre les deux guerres. Voir l'étude désormais classique de S. P. Rosenbaum, "The Philosophical Realism of Virginia Woolf ", dans S. P. Rosenbaum (ed.), English Literature and British Philosophy, Chicago, University of Chicago, 1971, et le livre d'Ann Banfield, The Phantom Table. Woolf, Fry, and the Epistemology of Modernism, Cambridge, Cambridge University Press, 2000; en français, J. Hintikka, "Virginia Woolf et notre connaissance du monde extérieur ", dans La vérité est-elle ineffable? Combas, L’Éclat, 1994, p. 73-94. 
Oxford, au même moment, Thomas Case et John Cook Wilson ont tous deux défendu une forme de "réalisme ». Ce dernier, par l'influence de ses étudiants, en premier lieu H. A. Prichard et H. W. B. Joseph, exercera une influence elle aussi déterminante sur la philosophie à Oxford. Les premières générations d'après-guerre dont faisaient partie Gilbert Ryle et $\mathrm{H}$. H. Price se tournèrent ainsi vers le "réalisme", et ceux-ci furent suivis dans les années trente par J. L. Austin, I. Berlin, J. O. Urmson, et al. ${ }^{61}$. Bref, au sortir de la Grande Guerre, l'idéalisme s'essouffle et n'attire plus les jeunes générations - les forces vives sont ailleurs, et le déclin final est alors inévitable, malgré le fait que des idéalistes sont encore nommés aux principales chaires à Oxford, jusqu'à Robin George Collingwood, qui sera «Waynflete Professor of Metaphysics» de 1935 à sa mort en 1943 (son successeur sera Gilbert Ryle).

Les raisons d'un tel déclin sont difficiles à cerner; les systèmes philosophiques ou les mouvements qui leur sont associés ne succombent pas, en général, aux seules «réfutations ». Dans le cas de l'idéalisme britannique, on peut à la rigueur parler d'ennui ${ }^{62}$ ou encore, avec David Stove, d'horror victorianorum $^{63}$; en effet, dès le début du siècle, c'est-à-dire lors du règne d'Edward VII, on cherchait à se démarquer de tout ce qui était symptomatique de l'ère victorienne. La politique fut en grande partie responsable de la popularité du mouvement idéaliste, mais elle semble aussi avoir été une cause de son déclin. Plusieurs ouvrages critiquent alors les idéalistes parce qu'ils font peu de cas de l'instinct et des facteurs irrationnels dans le comportement ${ }^{64}$. L'émergence, aux élections de 1906, d'un parti socialiste basé sur une classe sociale, le parti travailliste, renouvelle la pensée socialiste, et, pour plusieurs, dont le très influent Harold Laski ${ }^{65}$, la société est désormais reconnue comme étant composée de regroupements plus ou moins autonomes; l'État n'est donc plus perçu comme le lieu de l'accomplissement de l'individu ${ }^{66}$. La seconde

61. Voir la note 27 , ci-dessus.

62. G. Warnock, English Philosophy since 1900, Londres, Oxford University Press, 1958, p. $10-11$.

63. D. Stove, The Plato Cult, Oxford, Blackwell, 1991, p. 19.

64. Il n'est pas seulement question de l'influence de l'œuvre de Freud, mais aussi de l'étude de la notion d'instinct dans les disciplines neuves de cette époque, la psychologie et la sociologie. En psychologie, voir: B. Hart, The Psychology of Insanity, Cambridge, Cambridge University Press, 1912. W. H. Rivers, Instinct and the Unconscious; A Contribution to a Biological Theory of the Psycho-Neuroses, Cambridge, Cambridge University Press, 1920; et Psychology and Politics, and other Essays, K. Paul, Trench, Trubner \& Co., 1923. En sociologie: W. Trotter, "Herd Instinct and its Bearing on the Psychology of Civilised Man ", The Sociology Review, vol. 1 (1908), p. 227-248 et vol. 2 (1909), p. 36-54. La notion d' 'impulse » qu'utilisent ces auteurs occupe cependant une place centrale dans ce qui est fort probablement la contribution principale de Russell au libéralisme politique, ses conférences de 1916: The Principles of Social Reconstruction, Londres, Allen \& Unwin, 1916.

65. H. Laski, Studies in the Problem of Sovereignty, New Haven, Yale University Press, 1917; Authority in the Modern State, New Haven, Yale University Press, 1919.

66. Voir sur cette question l'article fort discuté à l'époque d'Ernest Barker, «The Discredited State: Thoughts on Politics before the War », The Political Quarterly, n. 5 (1915), p. 101-121. 
guerre des Boers (1899-1902) provoque au bout du compte une forte réaction anti-impérialiste, et les idéalistes sont attaqués pour leur défense de l'impérialisme par des libéraux comme John Hobson et Leonard Hobhouse (dont la sœur Emily avait dénoncé les camps de concentration $)^{67}$. Pour les socialistes et les libéraux, les idéalistes ne sont donc plus une référence; ces derniers sont en quelque sorte dépassés sur leur gauche; ils seront ensuite diabolisés. La Première Guerre mondiale provoque un fort sentiment nationaliste, antigermanique - on se rappellera par exemple les incidents de Louvain (dont l'incendie de la bibliothèque) et le scandale causé par l' "Aufruf an der Kulturwelt» où des universitaires allemands énoncèrent entre autres que leur culture devait sa survie au militarisme prussien —, les idéalistes seront à ce moment-là condamnés pour leurs sources allemandes par Dewey, Perry et Santayana ${ }^{68}$. R. B. Haldane, qui est Lord Chancellor, est accusé d'être un "espion hégélien» et doit démissionner en $1915^{69}$. Ce genre de sophisme de la culpabilité par association s'est perpétué après la Deuxième Guerre mondiale avec The Open Society and its Enemies, de Karl Popper, Hegel étant cette fois-ci accusé d'être un laquais du totalitarisme ${ }^{70}$.

Il faut aussi noter, avec Ryle ${ }^{71}$, une transformation de la profession de philosophe. Nous avons vu que l'étude de la philosophie était, au dix-neuvième siècle, intimement liée à celle de la théologie. Des auteurs tels que Strauss, Feuerbach, Renan, Newman et Colenso (un Anglais aujourd'hui oublié, évêque du Natal, qui a pourtant préfiguré la théologie de la libération) fournissaient matière à discussion. Cet état de choses disparaît entièrement après la Première Guerre mondiale - les philosophes ne proviennent alors plus du presbytère, ils ne méprisent plus les sciences naturelles, qu'ils étudient volontiers dans certains cas, et s'intéressent plutôt à des auteurs comme Marx, Frazer et Freud; à Cambridge, Ramsey est à la fois philosophe, mathématicien et économiste. Ce changement est aussi tangible chez certains idéalistes

67. Parmi les œuvres, voir L. T. Hobhouse, Democracy and Reaction, New York, Putnam, 1905 et J. A. Hobson, The Crisis of Liberalism, Londres, P. S. King \& Sons, 1909. Hobson publie en 1902 une critique de l'impérialisme, qu'il perçoit comme une recherche de nouveaux débouchés commerciaux par les capitalistes; cette idée est devenue largement répandue depuis, en particulier à travers son influence sur Lénine. Voir J. A. Hobson, Imperialism. A Study, $3^{\mathrm{e}}$ éd., Londres, Allen \& Unwin, 1938.

68. J. Dewey, German Philosophy and Politics, New York, H. Holt, 1915 ; R. B. Perry, The Present Conflict of Ideals. A Study of the Philosophical Background of the World War, New York, Longmans, 1922; G. Santayana, Egotism in German Philosophy, $2^{\mathrm{e}}$ éd., New York, Scribner, 1940. La première édition (1916) de ce livre de Santayana a été traduite en français: L'erreur de la philosophie allemande, Nouvelle librairie nationale, 1917.

69. J. Bradley, "Hegel in Britain...", op. cit., p. 176.

70. Sir K. Popper, The Open Society and its Enemies, 2 vol., Londres, Routledge \& Kegan Paul, 1945. Une critique plus nuancée, voir L. T. Hobhouse, The Metaphysical Theory of the State, Londre, Allen \& Unwin, 1918. Sur cette question, voir les textes recueillis dans W. Kaufmann (dir.), Hegel's Political Philosophy, New York, Atherton Press.

71. G. Ryle, «Introduction », dans The Revolution in Philosophy, Londres, MacMillan, 1956, p. 2-3. 
comme Collingwood, qui fut également un archéologue et un historien de renom. Par ailleurs, les théologiens, sous l'influence de Barth, s'éloignent rapidement de la philosophie ${ }^{72}$.

Entre ces deux époques, l'idéalisme britannique fut un mouvement suffisamment vaste pour engendrer ses propres dissensions internes - il n'y régna en réalité aucune véritable unité théorique. Seules sont constantes la référence aux grandes figures de l'idéalisme allemand et la critique obligée de la psychologie associationniste et de l'hédonisme utilitariste. Notons en passant qu'on doit, pour cette raison, aux idéalistes les premiers commentaires et les premières traductions en langue anglaise de Kant et de Hegel; les travaux de N. Kemp Smith, H. J. Paton sur Kant et de T. M. Knox et G. R. G. Mure sur Hegel sont au vingtième siècle un prolongement de cet engagement. (Soulignons cette particularité des lectures britanniques de Hegel qu'elles sont centrées, depuis The Secret of Hegel de Stirling et Hegel de Caird ${ }^{73}$, sur la Science de la logique ${ }^{74}$, plutôt que sur La phénoménologie de l'esprit, dont la «dialectique» n'eut jamais d'écho, les déboires politiques que nous avons mentionnés reportant tardivement l'attention sur la Philosophie du droit.) Dans ce qui suit, nous nous bornerons, en utilisant un classement en termes de générations, à introduire rapidement certaines distinctions qui permettent de mieux comprendre l'éventail des positions soutenues par les idéalistes britanniques. Il s'agira en fait essentiellement de clarifier le sens des trois paires d'adjectifs suivantes, dont on s'est servi pour qualifier l'idéalisme des Britanniques :

$$
\begin{gathered}
\text { subjectif - objectif } \\
\text { moniste - pluraliste } \\
\text { absolu - personnaliste }
\end{gathered}
$$

Comme nous l'avons vu, on peut distinguer, après une génération de précurseurs, dont faisaient partie Ferrier et Stirling (ainsi que d'autres figures non moins importantes comme John Grote à Cambridge ${ }^{75}$ ), une première

72. Il faut cependant noter les théologies idéalistes de William Temple, archevêque de Canterbury, et de F. R. Tennant, un étudiant de Ward. Voir, entre autres, W. Temple, Nature, Man and God, Londres MacMillan, 1934; F. R. Tennant, Philosophical Theology, 2 vol., Cambridge, Cambridge University Press, 1928-1930.

73. E. Caird, Hegel, Edimbourg, William Blackwood \& Sons, 1883.

74. J. M. E. McTaggart, A Commentary of Hegel's Logic, Cambridge, Cambridge University Press, 1910; G. R. G. Mure, A Study of Hegel's Logic, Oxford, Clarendon Press, 1950. De Errol Harris nous avons la traduction française de son commentaire de la Science de la logique: Lire la Logique de Hegel: commentaire de la Logique de Hegel, Lausanne, L'âge d'homme, 1987.

75. John Grote, frère de l'historien de la Grèce antique et membre du mouvement «radical », George Grote, a eu une influence profonde sur la philosophie à Cambridge, de William Whewell à Michael Oakeshott; celle-ci a été documentée de manière très détaillée dans J. Gibbins, John Grote, Cambridge University Press and the Development of Victorian Thought, Exeter, Imprint Academic, 2006. Grote a cependant été relégué aux oubliettes par ses ennemis Henry Sidgwick et Leslie Stephen. 
génération, celle de Green, d'Edward et de John Caird et de Bradley. Les étudiants de Green dans les années 1860-1880 forment ce que l'on peut appeler la seconde génération: Bosanquet, Mackenzie, Nettleship et Wallace. La figure principale de ces premières générations et du mouvement idéaliste dans son ensemble est sans conteste Bradley. Celui-ci raisonne dans Appearance and Reality à partir du principe de non-contradiction: ce qui est contradictoire ne peut pas être. Il dévoile alors les contradictions contenues dans notre conception ordinaire de la réalité, lorsque nous croyons que la réalité est composée d'objets indépendants les uns des autres et indépendants de l'expérience que nous en avons. Bradley soutient donc que le pluralisme et le réalisme ont des conséquences contradictoires et doivent être rejetés au profit de l'idée que la « réalité est une ${ }^{76}$ » et qu'elle est « expérience ${ }^{77}$ ». Bradley défend donc un «monisme » et un « idéalisme absolu », dont il faut noter, par ailleurs, qu'il n'est pas de même nature que celui de Hegel.

L'idéalisme de Bradley doit être soigneusement distingué de l'idéalisme subjectif. Selon une définition célèbre que l'on doit à Windelband, il faut comprendre par «idéalisme» la «dissolution (Auflösung) du monde de l'expérience dans le processus de la conscience ${ }^{78}$ ". Cette définition convient bien à l' «idéalisme subjectif» de Berkeley, de Fichte ou de Husserl, mais ne correspond pas à la position de Bradley. En effet, son monisme a pour conséquence que l'expérience n'est pas «expérience " pour un sujet qui serait une entité en dehors de l'expérience, car il n'y aurait alors plus de "monisme». Il faut donc qu'un des deux termes de la relation sujet-objet disparaisse, et Bradley énonce clairement que l'on "doit abandonner l'idée d'un soi qui serait de lui-même ou qui pourrait être réel ${ }^{79}$. En ce sens, même si l'expression n'a pas non plus le sens qu'elle a chez Hegel, il est plus approprié de parler, dans le cas de Bradley, d'un «idéalisme objectif ».

Le «monisme absolu» a été défendu par Bosanquet, qui reste malheureusement encore aujourd'hui dans l'ombre de Bradley. Cependant, ses apports sont nombreux. Pour ne prendre qu'un exemple, il semble être le premier à avoir identifié, dès sa première publication, la vérité avec l'expérience dans son intégralité en tant que système, identification qu'il reprendra sous le dicton "la vérité est le tout ${ }^{80}$. Bosanquet est ainsi à l'origine de la théorie de la vérité cohérence $^{81}$, implicite chez Bradley, et dont une version légèrement différente

76. F. H. Bradley, Appearance and Reality, $2^{\mathrm{e}}$ éd., Oxford, Clarendon Press, 1897, p. 460 et 462 .

77. Ibidem, p. 463, 475 et 489.

78. W. Windelband, A History of Philosophy, $2^{\mathrm{e}}$ éd., Londres, MacMillan, 1926, p. 569 n. 1. (Nous n'avons pas pu consulter l'original allemand.)

79. F. H. Bradley, Essays on Truth and Reality, Oxford, Clarendon Press, 1914, p. 326-327.

80. B. Bosanquet, "Logic as the Science of Knowledge », dans A. Seth \& R. B. Haldane (dir.), Essays in Philosophical Criticism, Londres, Longmans, Green and Co., 1883, p. 71 et 101; Logic or the Morphology of Knowledge, $2^{\mathrm{e}}$ éd., Oxford, Clarendon Press, 1911, vol. I, p. 3.

81. Ibidem, vol. II, chapitre ix, "Truth and Coherence ", p. 263-294 
sera défendue par la suite par H. H. Joachim, neveu du célèbre violoniste ${ }^{82}$. Mais le «monisme absolu» sera l'objet de plusieurs critiques, provenant d'une troisième génération d'idéalistes. Certains refuseront les conclusions «monistes» - ainsi McTaggart, pour qui la réalité est composée d'une pluralité d'âmes éternelles reliées entre elles par un amour mutuel ${ }^{83}$. Le psychologue James Ward conçoit, lui, la réalité comme une infinité de monades ${ }^{84}$. McTaggart est athée, et l'amour dont il parle n'est pas chrétien, mais, pour Ward, Dieu est nécessaire pour coordonner les monades. D'autres, à la suite d'Andrew Seth Pringle-Pattison, refusent le saut, effectué à la fois par Green et Bradley, de l'épistémologie à la métaphysique et affirment la nécessité de distinguer entre la thèse selon laquelle il ne peut y avoir de monde extérieur sans un sujet connaissant, et l'idée que matière et esprit sont inséparables. Pour Seth Pringle-Pattison, les néo-hégéliens, lorsqu'ils tentent comme Green d'unir l'individu à Dieu en retrouvant la copie de ce dernier dans la conscience de soi détruisent les deux termes de cette relation et privent l'individu de sa personnalité propre ${ }^{85}$. Contre le «monisme absolu» se développera donc, suivant Seth Pringle-Pattison et W. R. Sorley, un «idéalisme personnaliste ${ }^{86} »$. Parmi ces «personnalistes », F. C. S. Schiller tient une place à part, en intégrant des idées pragmatistes ${ }^{87}$. À ce courant, on peut également rattacher l'Américain Josiah Royce ${ }^{88}$.

82. H. H. Joachim, The Nature of Truth, Oxford, Clarendon Press, 1906, p. 76. Voir aussi H. H. Joachim, Logical Studies, Oxford, Clarendon Press, 1948. Joachim semble pencher dans The Nature of Truth, op. cit., p. 174-178, pour l'idée que la vérité cohérence doit ultimement s'appuyer sur une forme de vérité correspondance ou y aboutir, ce que Bosanquet récuse, Logic, op. cit., vol. II, p. 289. La position de Joachim n'est pas sans rappeler celle de Davidson dans "A Coherence Theory of Truth and Knowledge", dans Subjective, Intersubjective, Objective, Oxford, Clarendon Press, 2001, 137-153. L'article de N. Griffin dans ce volume fait le point sur l'apport de H. H. Joachim.

83. J. M. E. McTaggart, The Nature of Existence, Cambridge, Cambridge University Press, vol. 1, 1921 et vol. 2, 1927; et Studies in Hegelian Cosmology, Cambridge, Cambridge University Press, 1901. Sur la métaphysique de McTaggart, voir C. D. Broad, Examination of McTaggart's Philosophy, Cambridge, Cambridge University Press, volume I, 1933, volume II, Part I \& II, 1938; P. T. Geach Truth, Love and Immortality. An Introduction to McTaggart's Philosophy, Berkeley, University of California Press, 1979.

84. J. Ward, Naturalism and Agnosticism, Londres, A. \& C. Black, 1899; The Realm of Ends or Pluralism and Theism, Cambridge, Cambridge University Press, 1911.

85. A. Seth Pringle-Pattison, Hegelianism and Personality, Edimbourg, William Blackwood \& Sons, 1887, p. 62-64 et p. 221 sq.

86. H. Sturt (dir.), Personal Idealism: Philosophical Essays by Eight Members of the University of Oxford, Londres, Macmillan, 1902. Sur Seth et l'idéalisme personnaliste, voir J. Bradley, "Hegel in Britain... ", op. cit., p. 163-166.

87. F. S. C. Schiller, The Riddle of the Sphinx, Londres, Swan Sonnenschein \& Co., 1891; Humanism. Philosophical Essays, Londres, Macmillan, 1903; Studies in Humanism, Londres, Macmillan, 1907; Logic for Use, Londres, G. Bell \& Sons, 1929.

88. J. Royce, The Spirit of Modern Philosophy. An Essay in the Form of Lectures, Boston, Houghton Mifflin co., 1892; The World and the Individual, Londres, Macmillan, 1901; Lectures on Modern Idealism, New Haven, Yale University Press, 1919. 
Cette génération voit donc le mouvement idéaliste s'étioler - le "monisme absolu ", en particulier, ne recrute déjà plus personne ${ }^{89}-$, tandis qu'au même moment de nombreuses philosophies émergent en réaction expresse contre lui. Nous avons déjà évoqué la montée du "réalisme " en Grande-Bretagne, qui sera à l'origine des deux sources indépendantes de la philosophie analytique, une à Oxford, où enseigne Cook Wilson, et une autre à Cambridge, où étudient Russell et Moore. L'argument central de Russell, qui a joué un rôle fondamental dans la "réfutation» de l'idéalisme, porte sur ce qu'il appelle l'axiome des relations internes: "Toute relation est fondée dans la nature de ses termes ${ }^{90}$. » La cible en est la thèse de Bradley, selon qui «la réalité est une » parce que "la pluralité, considérée comme réelle, est contradictoire en soi », et ce parce qu'elle «implique des relations $»^{91}$. En d'autres termes, Bradley nie l'existence des relations: la réalité n'est qu'une chose, et il n'y a donc pas deux choses qui puissent être reliées. Une relation $R$ comme dans « $a$ a la relation $R$ à $b$ », ou $a R b$, ne serait en fait qu'une propriété $r$ d'un complexe $a b$, que l'on pourrait symboliser par: $(a b) r$. Toute relation serait donc réductible à une propriété d'un complexe. Pour Russell, cet axiome revient à réduire les relations à des prédicats (nous reviendrons làdessus), et à les analyser selon la distinction traditionnelle sujet-prédicat sous la forme « $a b$ est $r$ ». Or les relations transitives et asymétriques comme «est plus grand que» ne peuvent pas être analysées ainsi. Supposons que Jean mesure $1,80 \mathrm{~m}$, et Pierre, $1,90 \mathrm{~m}$. Si cela devait être analysé sous la forme $(a b) r$, alors il y aurait un complexe Jean-Pierre, qui ne pourrait pas être distingué de Pierre-Jean, qui aurait également la propriété «est plus grand que». Cette situation est catastrophique, à cause de l'asymétrie supposée de la relation: si Pierre est plus grand que Jean, alors Jean ne peut pas être plus grand que Pierre. Or, si l'on suit le Bradley de Russell, les deux totalités Jean-Pierre et Pierre-Jean, qui diffèrent entre elles seulement par l'ordre de leurs parties, satisferaient la propriété «être plus grand que».

Cette critique est en soi intéressante, mais il y a de bonnes raisons de douter qu'elle s'applique à Bradley, qui considérait l'idée qu'on puisse lui attribuer l'axiome des relations internes comme « ridicule» $(\text { ludicrous })^{92}$, ne

89. Seul Bosanquet continue une défense d'arrière-garde, par exemple dans The Value and Destiny of the Individual, Londres, MacMillan, 1913, pour ensuite tenter une conciliation dans son dernier livre, The Meeting of Extremes in Contemporary Philosophy, Londres, MacMillan, 1921.

90. B. Russell, Philosophical Essays, New York, Simon \& Schuster, 1968, 139-146; traduction française: Essais philosophiques, Paris, P.U.F., 1997, 195-203, et The Principles of

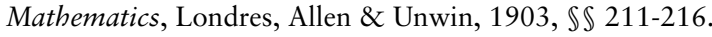

91. F. H. Bradley, Appearance and Reality, op. cit., p. 460.

92. F. H. Bradley, «Relations », Collected Essays, Oxford, Clarendon Press, 1935, p. 642. Il faut noter, cependant, la réaction tout à fait différente de Bosanquet, qui reprend à son compte l'axiome des relations internes dans Logic, op. cit., vol. II, p. 277. Malgré cela, il semble ridicule de faire reposer, comme Anthony Quinton, tout l'idéalisme britannique sur l'axiome des relations internes dans «Absolute Idealism», op. cit., p. 27-29. 
serait-ce que parce qu'il ne rejetait pas l'existence d'une distinction entre termes et relations, qui lui permettait par ailleurs de rendre compte de la distinction entre substance et accident. Dans son texte posthume, "Relations ", dernier acte de l'échange avec Russell, Bradley faisait remarquer que la différence entre termes et relations était basée sur une distinction entre la nature intrinsèque d'un objet et le fait qu'il entre en relation externe avec d'autres objets: cette distinction allait d'ailleurs à l'encontre de l'argument de la régression infinie que Bradley avait lui-même avancé (voir l'article de Nicholas Griffin dans ce volume $)^{93}$. Pour Bradley, toute relation était donc une «fiction de l'esprit », distinguant différents objets à l'intérieur d'une "réalité une »; l'erreur consistait à tenir les distinctions ainsi introduites pour des faits indépendants ${ }^{94}$. Russell se serait ainsi rendu coupable, dans sa critique de Bradley, du sophisme de l'ignoratio elenchi. Quoi qu'il en soit de cette question, que nous n'avons pas pour but de résoudre, pour des réalistes comme Russell ou Moore ${ }^{95}$, il existe des objets indépendants les uns des autres, qui ont entre eux des relations "externes", et le sujet est, dans la connaissance, en relation «externe» avec un objet indépendant de l'activité de connaissance. Cependant, ni Russell ni Moore n'ont réussi à donner un argument convaincant à l'appui de la réalité des relations. (Nous reviendrons sur ce point.)

La révolte de Moore et de Russell à Cambridge a un équivalent aux ÉtatsUnis, où domine la figure de William James, dont les travaux ont donné son essor au pragmatisme ${ }^{96}$, tout en influençant grandement les "nouveaux réalistes ", tels que E. B. Holt et R. B. Perry et, à leur suite, les «réalistes critiques » (A. Lovejoy, G. Santayana, R. W. Sellars). Tout comme Russell et Moore, ces «nouveaux réalistes» insistent sur la réalité des relations externes ${ }^{97}$.

93. F. H. Bradley, Appearance and Reality, op. cit., p. 27-28.

94. F. H. Bradley, The Principles of Logic, op. cit., vol. I, p. 96.

95. G. E. Moore, «External and Internal Relations », dans Philosophical Studies, Londres, Routledge \& Kegan Paul, 1922, p. 276-309.

96. Voir, entre autres, W. James, Pragmatism, op. cit.; A Pluralistic Universe, New York, Longmans, Green, and Co., 1909; Essays in Radical Empiricism, New York, Longmans, Green, and Co., 1912. James lui-même attribue à Peirce, qui la fait lui-même remonter à Alexander Bain, l'origine du pragmatisme. Voir W. James, Pragmatism, op. cit., p. 47-48; sur Bain, voir M. H. Fisch, "Alexander Bain and the Genealogy of Pragmatism ", dans Peirce, Semeiotic and Pragmatism, op. cit., 79-109. Un survol de la littérature au tournant du vingtième siècle montre cependant que Peirce a été largement ignoré par ses contemporains et que les discussions autour du pragmatisme portaient essentiellement sur l'œuvre de James, sans oublier celles de Dewey et de Schiller. (On se souviendra par ailleurs que Peirce s'était dissocié du "pragmatisme» de Dewey, James et Schiller, et qu'il avait introduit l'expression "pragmaticisme » pour désigner sa philosophie; C. S. Peirce, Collected Papers of Charles Sanders Peirce, C. Hartshorne \& P. Weiss (dir.), Cambridge MA, Harvard University Press, 6 vol., 1931-1958, 5.414 et 8.205.)

97. E. B. Holt, The New Realism: Cooperative Studies in Philosophy, New York, MacMillan, 1912. R. B. Perry, Present Philosophical Tendencies. A Critical Survey of Naturalism, Idealism, Pragmatism, and Realism together with a Synopsis of the Philosophy of William James, New York, Longmans, Green, and co., 1912. 
La popularité de ces nouvelles philosophies au début du siècle a pour résultat que l'idéalisme ne recrute déjà que peu ou prou de nouveaux adhérents parmi les Britanniques ou Américains éduqués à la veille de la Première Guerre mondiale. De cette quatrième génération, la seule figure importante est Collingwood, figure si peu orthodoxe qu'on ne le classe pas toujours parmi les idéalistes ${ }^{98}$. Ses cours sont très populaires, et on dit de lui qu'il aurait été le seul à être en mesure d'endiguer le raz-de-marée "analytique » qui déferlera sur Oxford et la Grande-Bretagne après la guerre, mais il meurt prématurément en 1943. On peut à la rigueur parler d'une cinquième et dernière génération d'idéalistes, de ceux qui furent éduqués entre les deux guerres, parmi lesquels Michael Oakeshott, qui étudie avec McTaggart à Cambridge au début des années vingt, mais ils resteront minoritaires, sans grande influence.

Avant de conclure cette partie, il faut insister sur la place centrale qu'occupe un philosophe allemand, trop souvent négligé aujourd'hui, dans les sources de l'idéalisme britannique. L'influence de Rudolf Hermann Lotze a été extrêmement forte sur la majorité des philosophes de cette école, de Green, Bosanquet et Bradley ${ }^{99}$, au psychologue James Ward et au théologien Hastings Rashdall ${ }^{100}$. À la fin du dix-neuvième siècle, à l'instigation de Green et Bosanquet, pas moins de neuf de ses livres ont été traduits en anglais; en France, seuls sa Métaphysique et un traité de physiologie ${ }^{101}$ firent l'objet d'une traduction à la même époque - tout récemment un chapitre de la Logique a été traduit ${ }^{102}$. Étant donné son influence, qui s'étend aussi à la théorie de la Geltung dans le néo-kantisme de l'école de Bade (Windelband, Rickert, Weber) et à Frege et Husserl, il faut regretter qu'il n'existe toujours aucune étude systématique de la philosophie de Lotze depuis celles, anciennes, d'Henry Jones, de George Santanaya, et de Philippe Devaux ${ }^{103}$.

Par ailleurs, il faut ajouter que l'idéalisme italien a joué un rôle important chez plusieurs philosophes britanniques, probablement à la suite de la

98. Par exemple, Jean Pucelle ne le mentionne même pas dans L'idéalisme en Angleterre, op. cit. Dans ses derniers écrits, Collingwood s'est éloigné de l'idéalisme, mais les premiers ouvrages restent très nettement idéalistes: R. G. Collingwood, Speculum Mentis, Oxford, Clarendon Press, 1924; Outlines of a Philosophy of Art, Oxford, Clarendon Press, 1925; Essay on Philosophical Method, Oxford, Clarendon Press, 1935; $2^{\mathrm{e}}$ éd., 2005.

99. Dans son premier livre, Bosanquet reproche justement à Bradley d'être trop proche de Lotze; B. Bosanquet, Knowledge and Reality. A Criticism of F. H. Bradley's Principles of logic, Londres, K. Paul Trench, 1885.

100. De Rashdall, éclipsé par Moore, il faut mentionner The Theory of Good and Evil. A Treatise on Moral Philosophy, Oxford, Clarendon Press, 1907.

101. R. H. Lotze, Métaphysique, Paris, Firmin-Didot, 1883 ; Principes généraux de psychologie physiologique, Paris, G. Baillère, 1881.

102. R. H. Lotze, «Le monde des idées », Philosophie, n. 91 (2006), p. 9-23.

103. H. Jones, A Critical Account of the Philosophy of Lotze. The Doctrine of Thought, New York, MacMillan, 1895; G. Santayana, Lotze's System of Philosophy, Bloomington IN, Indiana University Press, 1971; P. Devaux, Lotze et son influence sur la philosophie anglo-saxonne, op. cit. 
découverte fortuite de l'œuvre de Benedetto Croce par J. A. Smith avant la Première Guerre mondiale ${ }^{104}$. Figure mineure qui n'a guère publié, ce dernier s'est rangé au nombre des critiques de l'absolutisme de Bradley, sous l'influence de Croce et de Gentile ${ }^{105}$. Jusqu'à E. F. Carritt, H. J. Paton et Collingwood, Croce sera une référence à Oxford. Plusieurs de ses livres ont été traduits, dont trois par Collingwood, mais l'étendue de l'influence que Croce exerça sur ce dernier est encore mal comprise de nos jours. Cette question est abordée en partie dans le texte de Chinatsu Kobayashi, qui aborde les critiques de Bosanquet et de Collingwood à l'endroit de la philosophie de l'art de Croce.

\section{Peut-on parler d'une actualité de l'idéalisme britannique?}

The Hegelian philosophy has to die that it may live.

EDWARD CAIRD

De la doctrine de Shadworth Hodgson, figure mineure s'il en est, mais prolifique de la philosophie britannique de la deuxième moitié du dix-neuvième siècle, l'historien allemand Metz disait avec dérision que «le vaste cimetière de l'histoire de la philosophie l'a accueilli et lui a assigné une place d'honneur ${ }^{106}$. On peut se demander s'il n'en est pas ainsi pour les grandes figures de l'idéalisme britannique que furent Green, McTaggart, Bradley, Bosanquet ou Collingwood. En effet, pourquoi les étudier de nouveau, après les avoir ignorés pendant tant de décennies? Outre la curiosité de l'historien, il y a plusieurs raisons à cela. Premièrement, il faut insister sur la valeur intrinsèque des grandes œuvres que nous ont légués ces idéalistes. Par exemple, Bradley est de loin le métaphysicien le plus remarquable d'un dix-neuvième siècle britannique déjà fort riche. On peut saluer dans son œuvre, écrite dans un style que T. S. Eliot décrivait comme "parfait ${ }^{107}$, un véritable monument et ce, même si on croit possible de desceller quelques pierres dans les fondements de l'édifice pour le faire s'écrouler. Si Bradley n'est guère lu aujourd'hui, c'est sans doute parce qu'il a avancé ses conclusions de façon rationnelle en

104. J. A. Smith, "Philosophy as the Development of the Notion and Reality of SelfConsciousness ", dans J. H. Muirhead (dir.), Contemporary British Philosophy, sec. series, Londres, Allen \& Unwin, 1924, p. 230-231.

105. Ibidem, p. 232-233.

106. R. Metz, A Hundred Years of British Philosophy, op. cit., p. 483.

107. T. S. Eliot, "Francis Herbert Bradley", dans Selected Essays, 1917-1932, New York, Harcourt \& Brace, 1932, p. 359. T. S. Eliot a en outre rédigé une thèse de doctorat sur Bradley à Harvard en 1916, Knowledge and Experience in the Philosophy of F. H. Bradley (New York, Farrar, Strauss \& Company, 1964). Il y reconnaît même une influence de Bradley sur sa prose (T. S. Eliot, Knowledge and Experience, op. cit., p. 11). L'influence de la philosophie de Bradley se fait sentir entre autres sur le contenu des Four Quartets. Toujours au chapitre de la présence de Bradley dans la littérature, signalons les nombreuses références à Appearance and Reality dans l'œuvre de l'écrivain argentin Jorge Luis Borges, par exemple dans "Avatars de la tortue» (Enquêtes 1937-1952, Gallimard, 1957). 
réfutant, comme les Éléates (Parménide et Zénon viennent à l'esprit ${ }^{108}$ ), les conclusions adverses par la démonstration du fait que ces dernières ont des conséquences contradictoires. Pour cette raison, Russell avait le plus grand respect pour les œuvres de Bradley, malgré un désaccord complet sur ses conclusions ${ }^{109}$. On peut soupçonner que ceux qui, de nos jours, seraient susceptibles de les approuver ne ressentent plus le besoin de fonder leurs opinions sur des arguments: la lecture de Bradley pourrait ainsi leur paraitre fastidieuse.

Deuxièmement, il faut noter que les doctrines idéalistes ne sont pas toutes déjà enterrées au cimetière de l'histoire de la philosophie. En fait, beaucoup de leurs thèses sont encore discutées et forment même dans certains cas l'origine de pans entiers de la philosophie analytique. Les exemples ne peuvent certes pas être multipliés, mais en voici quelques-uns:

- La preuve de l'irréalité du temps de McTaggart est depuis toujours au cœur des réflexions analytiques sur le temps ${ }^{110}$.

- La théorie des explications rationnelles en histoire, de Collingwood, après de multiples méprises sur son sens, comme explication de rechange au modèle des lois englobantes (covering laws) de Hempel ${ }^{111}$.

108. Cette impression est fréquente, par exemple, J. H. Muirhead, "Past and Present ...", op. cit., p. 318; F. Houang, Le néo-hégélianisme en Angleterre, op. cit., p. 12, note 1; J. Skorupski, English-Language Philosophy, op. cit., p. 89.

109. B. Russell, «Philosophy in the Twentieth Century ", réimprimé dans The Collected Papers of Bertrand Russell, vol. 9, Londres, Unwin Hyman, 1983, p. 452.

110. J. M. E. McTaggart, "The Unreality of Time», dans Philosophical Studies, Londres, Edward Arnold, 1934, pp. 110-131; The Nature of Existence, Cambridge, Cambridge University Press, vol. 2, 1927, $\mathbb{S}$ S303-351. L'article est traduit dans S. Bourgeois-Gironde, McTaggart: temps, éternité, immortalité, Combas, Éditions de l'Éclat, 2000. La littérature en anglais sur ce sujet est immense, nous ne mentionnerons que l'important article de M. A. E. Dummett, "A Defense of McTaggart's Proof of the Unreality of Time", dans Truth and other Enigmas, Londres, Duckworth, 1978, p. 351-357. En français, on trouve dans la littérature secondaire, outre l'étude de Bourgeois-Gironde, F. Cayla, «La preuve de McTaggart et l'irréalité du temps », dans J.-M. Vienne (dir.), Philosophie analytique et histoire de la philosophie, Paris, Vrin, 1997, p. 303-313; M. Marion, "La "preuve” de McTaggart ", Carrefour, vol. 22, n. 2, 2000, p. 83103. On notera par ailleurs la discussion de McTaggart et de Dummett par l'écrivain français Jacques Roubaud, membre de l'OULIPO. Voir J. Roubaud, L'abominable tisonnier de John McTaggart Ellis McTaggart et autres vies plus ou moins brèves, Paris, Éditions du Seuil, 1997.

111. La première reprise des idées de Collingwood contre l'approche positiviste de Dray se trouve dans W. H. Dray, Laws and Explanations in History, Oxford, Clarendon Press, 1957. Dray a publié son interprétation de Collingwood à la fin de sa carrière, dans W. H. Dray, History as Re-Enactment, Oxford, Clarendon Press, 1995. Parmi les ouvrages dédiés à la philosophie de l'histoire de Collingwood, voir: H. Saari, Re-enactment: A Study in R. G. Collingwood's Philosophy of History, Åbo, Åbo Akademi, 1984; P. Skagestad, Making Sense of History. The Philosophies of Popper and Collingwood, Oslo, Universtetsfortlaget, 1975; W. J. van der Dussen, History as a Science: The Philosophy of R.G. Collingwood, The Hague, Martinus Nijhoff, 1981. En français, voir C. Kobayashi \& M. Marion, "La philosophie de l'histoire de Collingwood: rationalité, objectivité et anti-réalisme ", dans C. Nadeau (dir.), La philosophie de l'histoire $a u X^{e}$ siècle. Hommages offerts à Maurice Lagueux, Québec, Presses de l'Université Laval, 2007, p. 119-164. 
- Bosanquet, Joachim et Blanshard restent des incontournables en ce qui concerne une des théories de la vérité, certes aujourd'hui peu populaire, celle de la vérité comme cohérence ${ }^{112}$.

Cet héritage est certes fragmentaire, mais ce constat ne dispense personne d'ignorer les sources.

La théorie de la vérité cohérence est au centre de la contribution à ce numéro du grand spécialiste de Russell, Nicholas Griffin, qui porte sur les relations entre Russell et Joachim. L'argument «standard" contre la vérité cohérence qu'on doit à Russell fonctionne comme ceci : je peux formuler un énoncé manifestement faux, comme "L'évêque Stubbs a été pendu pour meurtre ", et concevoir un nombre indéfini d'ensembles d'énoncés dans lesquels il peut s'insérer - ce qui le rend vrai - , mais où ne le peut pas celui, pourtant vrai, selon lequel il est mort dans son lit ${ }^{113}$. Nicholas Rescher a voulu contourner cette objection en considérant plutôt la cohérence comme un «critère» nous permettant de parvenir à la vérité ${ }^{114}$; d'autres, comme Ralph Walker, ont fait valoir que les idéalistes ne parlaient pas de cohérence relativement à un nombre indéfini d'ensembles arbitraires d'énoncés, mais plutôt relativement à un ensemble bien précis, par exemple, l'Absolu ou celui de la science à la fin de l'enquête ${ }^{115}$.

Troisièmement, on notera une tendance récente en philosophie analytique, plus prononcée dans un domaine comme la philosophie des sciences, à l'étude de sa propre histoire. Le renouveau des études sur l'idéalisme britannique (en particulier sur Bradley et sur Collingwood) que l'on constate depuis une dizaine d'années n'est pas sans rapport avec cette tendance générale. Selon une image répandue, les philosophes analytiques sont censés lire un dialogue de Platon comme s'il avait été publié l'année précédente ${ }^{116}$. Il n'est donc pas étonnant qu'ils se soient peu préoccupés de leur propre histoire et, lorsque c'est le cas, qu'ils ne le fassent pas de manière historiciste ${ }^{117}$. Cette approche n'est pas sans vertu, mais il faut signaler un nombre croissant d'études qui cherchent à reconstruire la signification des doctrines analytiques en élaborant un contexte d'explication à l'intérieur duquel elles prendraient leur

112. B. Bosanquet, Logic, op. cit.; H. H. Joachim, The Nature of Truth, op. cit., et Logical Studies, op. cit.; B. Blanshard, The Nature of Thought, Londres, Allen and Unwin, 1939.

113. B. Russell, Philosophical Essays, op. cit., p. 136; trad. française, op. cit., p. 193.

114. N. Rescher, The Coherence Theory of Truth, Oxford, Oxford University Press, 1973.

115. Voir R. C. S. Walker, The Coherence Theory of Truth: Realism, Anti-Realism, Idealism, Londres, Routledge, 1989; C. Wright, «Critical Study: Ralph C. S. Walker, The Coherence Theory of Truth: Realism, Anti-Realism, Idealism ", Synthese, vol. 103, 1995, p. 279302; J. O. Young, "A Defence of the Coherence Theory of Truth", Journal of Philosophical Research, vol. 26, 2001, p. 89-101

116. Voir sur cette question les contributions de Jonathan Barnes et de Hidé Ishiguro au numéro spécial de la revue Critique sur Les philosophes anglo-saxons par eux-mêmes (numéro 399-400, août/septembre 1980).

117. L'exemple le plus pertinent étant les excellents volumes de Scott Soames, Philosophical Analysis in the Twentieth Century, 2 vol., Princeton, Princeton University Press, 2003. 
sens, un peu comme un coup dans un jeu peut être expliqué et prend sa signification à partir de son contexte (et des règles de ce jeu). Cette approche de l'histoire des idées trouve paradoxalement son origine dans l'œuvre de... Collingwood ${ }^{118}$; elle s'oppose entre autres à l'approche de l'histoire de la philosophie popularisée en France par Martial Guéroult, qui se limite à l'examen interne de l'œuvre, supposée contenir en elle-même les ressorts de son intelligibilité. Dans ce cadre, une bonne compréhension des œuvres des idéalistes britanniques est essentielle à la reconstruction du contexte d'origine de la philosophie analytique. Les études de Peter Hylton et de Nicholas Griffin sur la philosophie de Russell sont de très bons exemples de la voie à suivre ${ }^{119}$. Ainsi, si les critiques que Russell adresse à Bradley sont restées célèbres, peu se souviennent des objections que Bradley a élevées à l'encontre des thèses russelliennes, à la fois dans ses articles ${ }^{120}$ et dans sa correspondance ${ }^{121}$. Or ces critiques ont eu des répercussions sur le développement de la pensée russellienne elle-même. Cela vaut tout particulièrement pour l'argument de la régression à l'infini de Bradley, d'une grande importance dans son argumentation contre le pluralisme dans Appearance and Reality, et selon lequel toute relation, si elle existe comme terme indépendant des termes qu'elle relie, doit elle-même être reliée à chacun des termes par une autre relation, qui doit elle-même être, pour la même raison, reliée à chacun de ses termes par une autre relation, et ainsi de suite, ad infinitum ${ }^{122}$. Russell a critiqué de nombreuses fois cet argument, qu'il croit lié chez Bradley à une thèse selon laquelle il n'y aurait qu'une seule forme propositionnelle, celle qui a trait à la forme

118. Cette approche "collingwoodienne» a été discutée pour la première fois par l'historien de la philosophie, John Passmore dans "The Idea of a History of Philosophy ", History and Theory, Beiheft 5, vol. 5, 1965, p. 1-32. Elle fut reprise en histoire des idées par l'école de Cambridge, dont Quentin Skinner est le représentant le mieux connu; voir, entre autres, Q. Skinner, «The Rise of, Challenge to and Prospects for a Collingwoodian Approach to the History of Political Thought ", dans D. Castiglione \& I. Hampsher-Monk (dir.), The History of Political Thought in National Context, Cambridge, Cambridge University Press, 2001, p. 175-188. Cette approche a aussi été mise en application par l'historien de l'art Michael Baxandall, dans Patterns of Intention. On the Historical Explanation of Pictures, New Haven, Yale University Press, 1985. Son analyse du Baptême du Christ de Piero della Franscesca au chapitre IV est exemplaire.

119. P. Hylton, Russell, Idealism and the Emergence of Analytic Philosophy, Oxford, Clarendon Press, 1990; N. Griffin, Russell's Idealist Apprenticeship, Oxford, Oxford University Press, 1990. Pour une critique de l'usage que ces deux études font des idéalistes, voir l'ouvrage récent de S. Candlish, The Russell/Bradley Dispute and its Significance for Twentieth Century Philosophy, Basingstoke, Palgrave Macmillan, 2006; sur Bradley et Russell, voir également G. Stevens, The Russellian Origins of Analytical Philosophy - Bertrand Russell and the Unity of the Proposition, Londres, Routledge, 2005; sur les relations de Russell à l'idéalisme et aux diverses sortes de réalisme, voir également l'ouvrage de O. Nasim, Bertrand Russell And The Edwardian Philosophers: Constructing The World, Houndmills, Palgrave MacMillan, 2008.

120. Ces textes sont recueillis dans F. H. Bradley, Essays on Truth and Reality, op. cit.

121. F. H. Bradley, Selected Correspondence January 1905-June 1924, Bristol, Thoemmes Press, 1999.

122. F. H. Bradley, Appearance and Reality, op. cit., p. 27-28. 
sujet-prédicat ${ }^{123}$. Or, dans la critique qu'il fait lui-même de Russell, Bradley prend précisément pour cible la notion de forme (ou d'unité) propositionnelle. Russell distinguait dans les Principles of Mathematics, la notion d' "agrégat ", un tout composé de parties, de celle d' "unité », qui est celle d'un tout ne se réduisant pas à ses parties ${ }^{124}$. Si les classes sont le prototype des agrégats, les propositions sont le paradigme des unités - en effet, pour Russell, une proposition ne s'identifie pas à l'ensemble de ses constituants, car il est impossible de reconstituer à partir d'eux l'unité qu'est la proposition. Russell prétendait que c'est la relation (ou comme il le disait: le "verbe»), en tant qu'elle «relie », qui effectue le lien entre les constituants; la relation était donc, pour lui, un terme comme un autre, et plus qu'un terme, puisqu'il lui revenait d'assurer la liaison entre elles et les autres constituants. Bien évidemment, l'occasion était trop belle pour Bradley, qui attaqua son adversaire à de multiples reprises sur ce point. Russell admit le bien-fondé de la critique; il écrivit ainsi à Bradley en janvier 1914: "Je reconnais pleinement l'importance vitale des questions que vous posez, particulièrement celle qui concerne les « unités »; je reconnais comme mon devoir de parvenir à une réponse et, si je ne peux pas, d'en rechercher une aussi longtemps que je vivrai ${ }^{125}$.» L'aveu de Russell ne doit pas être pris à la légère; selon Graham Stevens, la question de la nature des unités constituerait bien le fil directeur de l'ensemble de la pensée russellienne, des Principles of Mathematics (1903) à Human Knowledge (1948) et des motifs recteurs de la première philosophie de Wittgenstein ${ }^{126}$. Bref, même d'un point de vue russellien, la confrontation avec Bradley ne s'arrête pas en 1903 ou en 1906 (date des articles sur la théorie de la vérité de Joachim) — l'idée que la forme d' « unité dans la différence» requise pour penser la proposition et la relation échappe à la raison analytique discursive continuera de hanter Russell.

Le texte de Mathieu Marion vient apporter dans ce contexte un nouvel élément au dossier des sources idéalistes de la philosophie analytique, celui de l'anti-psychologisme de Bradley, qui est une source plus directe pour les Britanniques que celles de Frege, de Husserl, ou de Cohen. Ce genre d'argument vaut tout autant pour le pragmatisme américain, dans la mesure où l'idéalisme absolu de Bradley et de Royce fit partie du contexte d'origine des œuvres de William James et de John Dewey ${ }^{127}$. Pensons aux parallèles assez flagrants

123. Voir B. Russell, A Critical Exposition of the Philosophy of Leibniz, Londres, Cambridge, Cambridge University Press, 1900, p. 15; The Principles of Mathematics, op. cit., \$99; Philosophical Essays, op. cit. p. 142; trad. française, p. 198. Il existe bien sûr de nombreuses autres répliques à Bradley; voir R. Gaskin, "Bradley's Regress, the Copula and the Unity of the Proposition », Philosophical Quarterly, vol. 45, 1995, p. 161-180.

124. B. Russell, The Principles of Mathematics, op. cit., $\mathbb{\$} \$ 136-139$.

125. Ibid. p. 181.

126. Voir G. Stevens, The Russellian Origins of Analytical Philosophy, op. cit.

127. Voir, par exemple, T. L. S. Sprigge, James and Bradley: American Truth and British Reality. Chicago, Open Court, 1993. Voir aussi Russell Goodman, American Philosophy and the Romantic Tradition, Cambridge, Cambridge University Press, 1991. 
entre le concept d' «expérience» sur lequel Bradley fonde son monisme, et celui d' "expérience pure ", sur lequel James fonde son "monisme neutre» dans «Does Consciousness Exist?» et "A World of Pure Experience " ${ }^{128}$; James a lui-même tenté sans succès un rapprochement avec Bradley dans leur correspondance ${ }^{129}$.

Par ricochet, il n'est pas impossible que ce regain d'intérêt pour la confrontation entre les idéalistes britanniques, d'un côté, et les premiers philosophes analytiques ou les premiers pragmatistes, de l'autre, puisse enrichir ou modifier l'image que l'on se fait habituellement de l'idéalisme. D'une part, le retour sur le devant de la scène d'un philosophe comme Hermann Lotze permet de souligner l'existence de continuités entre les différentes «Logik» des idéalistes allemands (celle de Hegel au premier chef), celle des idéalistes britanniques (Bradley et Bosanquet essentiellement), et la nouvelle logique de Russell et de Frege (puisque Frege a été influencé par Lotze ${ }^{130}$ ). Le récent ouvrage de James Allard permet ainsi de suivre les inflexions et les mutations subies par des thèmes originairement hégéliens ou kantiens, celui du système et du statut de la disjonction, par exemple ${ }^{131}$. L'étude de Sébastien Gandon sur la critique de Bradley par Royce et Russell fait aussi ressortir le rôle de Lotze dans ce contexte. D'autre part, il n'est pas certain que, comme on l'entend souvent dire, la philosophie des sciences constitue le parent pauvre de l'idéalisme, qu'il soit décliné dans sa version britannique ou allemande. Les idéalistes n'étaient pas réfractaires aux nouvelles avancées scientifiques, comme on aurait fâcheusement tendance à le croire; nous avons vu, par exemple, que certains d'entre eux ont même tenté de conjuguer la philosophie de Hegel à l'évolutionnisme de Darwin ${ }^{132}$. Ici encore, la figure de Lotze, mathématicien, physicien et psychologue, nous conduit à reconsidérer quelques préjugés. L'élaboration des calculs géométriques, qui auront un grand succès en Angleterre, s'est ainsi effectuée, chez Grassmann, dans un cadre explicitement idéaliste ${ }^{133}$. En nous limitant à l'Angleterre, il ne nous faut pas oublier que l'idéalisme forme le contexte du premier livre d'épistémologie de Russell,

128. W. James, Essays in Radical Empiricism, op. cit., chapitre I et II.

129. Sur celle-ci, voir T. L. S. Sprigge, James and Bradley, op. cit., p. 585-595.

130. Cette influence, qui a fait il y a quelques années l'objet d'une controverse (entre M. Dummett et H. Sluga), avait pourtant été déjà remarquée par Philippe Devaux dans Lotze et son influence sur la philosophie anglo-saxonne, op. cit., p. 23.

131. Voir J. W. Allard, The Logical Foundations of Bradley's Metaphysics: Judgment, Inference, and Truth, op. cit.; notamment au chapitre 5. Voir aussi sur la question de la négation, J.-P. Narboux, "Négation, contrariété et contradiction: sur la théorie éliminativiste de la négation dans l'idéalisme anglais ", op. cit.

132. Voir note 31, ci-dessus. Bien entendu, les sciences de la vie constituent également un terrain où la dispute est particulièrement vive. Citons l'œuvre classique de D'Arcy Wentworth Thompson, On Growth and Form (Cambridge, Cambridge University Press, 1917), qui s'inscrit clairement dans une veine anti-darwinienne et n'est pas sans connexion avec l'idéalisme.

133. Voir notamment A. C. Lewis, «H. Grassmann's, 1844, Ausdehnungslehre and Schleiermacher's 'Dialektik' », Annal of Science, vol. 34, 1977, p. 103-162. 
An Essay on the Foundations of Geometry (1897), où culmine la tradition anglaise de géométrie descriptive (Arthur Cayley, W. K. Clifford, etc.) ${ }^{134}$. Rappelons aussi que Peter Guthrie Tait liait sa classification des nœuds (qui est à l'origine d'un chapitre de la topologie ${ }^{135}$ ), élaborée dans le cadre de la théorie anti-matérialiste des atomes vortex de Thomson, à une défense du dogme chrétien de l'immortalité de l'âme. Le livre dans lequel il expose ses conceptions, Unseen Universe ${ }^{136}$, écrit en collaboration avec le chimiste Balfour Stewart, connut un très grand succès. Il ne s'agit pas simplement de rappeler par là les étranges croyances des grands savants de l'époque, mais de montrer que certaines thèses idéalistes ont pu s'incarner dans des formes scientifiques ou fournir le cadre d'interprétation naturel de pratiques scientifiques déjà existantes. Dans le même ordre d'idées, le rôle historique que l'on attribue à l'économiste Alfred Marshall, un des fondateurs de la théorie néoclassique qui a dominé cette discipline au vingtième siècle, fait passer sous silence ses positions philosophiques et éthiques sur l'économie, lesquelles étaient en fait apparentées à celles de Green, à l'opposé de celles de ses contemporains W. S. Jevons, Henry Sidgwick et F. Y. Edgeworth, qui établirent des liens aujourd'hui bien connus avec l'utilitarisme ${ }^{137}$.

La dernière raison d'étudier de nouveau les travaux des idéalistes britanniques est qu'une meilleure compréhension de leur pensée pourrait réactualiser leurs œuvres. Les travaux de Robert Brandom et de John McDowell montrent que l'époque où l'œuvre de Hegel était tenue comme un ramassis de non-sens est révolue ${ }^{138}$. Les livres des idéalistes britanniques ont été mis de côté à la suite d'une critique trop souvent facile et fort injuste parce qu'il en

134. B. Russell, An Essay on the Foundations of Geometry, Cambridge, Cambridge University Press, 1897. Sur Russell et la tradition anglaise, voir J. L. Richards, Mathematical Visions. The Pursuit of Geometry in Victorian England, San Diego, Academic Press, 1988.

135. Sur ce point, voir M. Epple, "Topology, Matter and Space, I. Topological Notions in 19th Century Natural Philosophy ", Archive for History of Exact Sciences, 52, 1998, p. 297-392.

136. P. G. Tait \& B. Stevens, Unseen Universe or Physical Speculations of a Future Universe, Macmillan Company, 1875.

137. Voir C. J. Dewey, "'Cambridge Idealism': Utilitarian Revisionists in Late NineteenthCentury Cambridge », History Journal, vol. 17, 1974, p. 63-78.

138. Voir en particulier R. Brandom, Tales of the Mighty Dead, Cambridge MA, Harvard University Press, 2002, chap. 6-7 (le chapitre 7 est paru en français: "Quelques thèmes pragmatistes dans l'idéalisme de Hegel: Négociation et administration dans la conception hégélienne de la structure et du contenu des normes conceptuelles ", Philosophiques, vol. 27, n. 2, 2000, p. 231-261) et J. McDowell, Mind and World, Cambridge MA, Harvard University Press, 1994 (traduction française: L'esprit et le monde, Paris, Vrin, 2007) et Having the World in View. Essays on Kant, Hegel, and Sellars, Cambridge MA., Harvard University Press, 2009. Pour d'autres articles en français, y compris de Brandom et de McDowell, voir aussi D. Perinetti \& M.-A. Ricard, La Phénoménologie de l'Esprit de Hegel: lectures contemporaines, Paris, Presses Universitaires de France, 2009, et le numéro spécial Hegel pragmatiste? de la revue Philosophie, numéro 99, 2008. Bien sûr, ces lectures analytiques sont controversées, mais dans le cadre de cet article il importe au moins de noter qu'elles sont essentiellement basées sur La phénoménologie de l'esprit et non sur la Science de la logique, qui joua un rôle si important il y a 150 ans. 
a résulté un ensemble de stéréotypes qui condamnent stupidement l'accès aux œuvres. C'est sans doute le cas de la critique de Bradley par Russell ${ }^{139}$, dont avons vu qu'on ne peut pas dire qu'il l'ait «réfuté » ou même qu'il ait donné un argument en faveur de la réalité des relations ${ }^{140}$. Nous pouvons ici appeler comme témoin nul autre que Donald Davidson, qui a reconnu dans Truth and Predication que les philosophes n'ont fait depuis que peu de progrès sur la question de l'unité de la proposition et de la prédication, la plupart ne reconnaissant même pas l'existence d'un problème ${ }^{141}$. Pourtant, selon Davidson, dans ce débat «Bradley a gagné, mais... Russell était du bon côté ${ }^{142}$.

Toujours au sujet de Bradley, on a aussi longtemps soutenu, à la suite de Russell1 ${ }^{143}$, qu'il avait défendu une conception de la vérité cohérence; on reconnaît aujourd'hui, grâce aux travaux de Thomas Baldwin et de Stewart Candlish ${ }^{144}$, qu'il fut plutôt un précurseur de la théorie de la vérité identité, c'est-à-dire la thèse selon laquelle une proposition est vraie dans la mesure où elle est identique à son vérifacteur (truth-maker $)^{145}$. On trouverait en John McDowell un défenseur contemporain de cette approche ${ }^{146}$. Certes, l'attribution d'une telle théorie à Bradley est controversée et, de surcroît, elle doit encore être plus solidement établie ${ }^{147}$, mais le fait même qu'une telle comparaison ait pu être faite indique déjà qu'une lecture des œuvres de Bradley libre de préjugés peut rapporter des dividendes.

139. Voir, S. Candlish, The Russell/Bradley Dispute, op. cit., chapitre 1, pour un ensemble de stéréotypes dérivés des critiques de Bradley par Russell.

140. Voir plus haut. Sur cette question, voir S. Candlish, The Russell/Bradley Dispute, op. cit., chapitre 6.

141. D. Davidson, Truth and Predication, Cambridge MA, Harvard University Press, 2005, p. 94. Strawson est l'exception.

142. Ibidem, p. 106. Certes, la solution que privilégie Davidson (comme on pouvait s'y attendre: une glose sur la définition de la vérité par Tarski) n'est pas dans la lignée de celle de Bradley.

143. Dans son célèbre article «La théorie moniste de la vérité », Russell critique Joachim, mais sème la confusion en citant surtout Bradley; B. Russell, Philosophical Essays, op. cit., p. 131-146; trad. française, op. cit., 185-203. Pour un exemple de ce genre de confusion, voir S. Haack, Philosophy of Logics, Cambridge, Cambridge University Press, 1978, p. 94-97.

144. S. Candlish, "The Truth about F. H. Bradley", Mind, vol. 97, 1989, p. 331-348; T. Baldwin, «The Identity Theory of Truth », Mind, vol. 100, 1991, p. 35-52.

145. S. Candlish, "The Identity Theory of Truth", dans E. N. Zalta (dir.), The Stanford Encyclopedia of Philosophy [en ligne: http://plato.stanford.edu/entries/truth-identity]. 160-165.

146. J. Dodd, "McDowell and Identity Theories of Truth ", Analysis, vol. 55, 1995,

147. Candlish a donné par la suite des raisons de croire que l'attribution de la théorie de l'identité à Bradley n'est pas tout à fait exacte dans S. Candlish, "Resurrecting the Identity Theory of Truth ", Bradley Studies, vol. 1, 1995, 116-124. Pour une défense de la théorie de l'identité: S. Candlish, "A Prolegomenon to an Identity Theory", Philosophy, vol. 74, 1999, 199-220 ; J. C. Beall, "On the Identity Theory of Truth ", Philosophy, vol. 75, 2000, 127-130. Voir aussi, sur l'ensemble de ces questions, S. Candlish, The Russell/Bradley Dispute, op. cit., chapitre 4. L'article de N. Griffin dans ce volume revient sur ces questions. 
Pour toutes ces raisons, la philosophie de Bradley est l'objet d'un regain d'intérêt depuis une vingtaine d'années ${ }^{148}$. Il en va de même, dans une moindre mesure, pour la philosophie de Collingwood ${ }^{149}$. Ainsi, dans sa contribution à ce numéro, Laurent Jaffro montre la pertinence des critiques de Collingwood à l'endroit du recours de Moore au sens commun contre le soi-disant obscurantisme des idéalistes. L'auteur de Collingwood and the Metaphysics of Experience ${ }^{150}$, Giuseppina D'Oro discute de son côté les thèses de Collingwood en relation avec la question tout à fait contemporaine du fossé explicatif.

Nous avons déjà indiqué la pertinence de l'approche de Collingwood en histoire des idées; sa philosophie est d'ailleurs surtout restée vivante chez les politologues et les historiens britanniques de la pensée politique grâce à son New Leviathan, le seul ouvrage d'ailleurs traduit en français ${ }^{151}$. Ryle a rejeté Collingwood du revers de la main en le qualifiant d' «idéaliste» — ce qui ne lui a guère $\mathrm{plu}^{152}$ - pour ensuite reconnaître que Collingwood avait préfiguré... sa propre critique du dualisme cartésien, sur laquelle il a bâti toute sa

148. J. W. Allard, The Logical Foundations of Bradley's Metaphysics: Judgment, Inference, and Truth, op. cit.; P. Basile, Experience and Relations: An Examination of F.H. Bradley's Conception of Reality, Berne, Paul Haupt, 1999; J. Bradley (dir.), Philosophy After F.H. Bradley, Bristol, Thoemmes Press, 1996, S. Candlish, The Russell/Bradley Dispute, op. cit.; W. Mander, An Introduction to Bradley's Metaphysics, Oxford: Clarendon Press, 1994; W. Mander (dir.), Perspectives on the Logic and Metaphysics of F.H. Bradley, Bristol, Thoemmes Press, 1996; A. Manser, Bradley's Logic, Oxford, Blackwell, 1983; A. Manser et G. Stock (dir.), The Philosophy of F.H. Bradley, op. cit.; T. L. S. Sprigge, James and Bradley, op. cit.; G. Stock, Appearance versus Reality, op. cit. Signalons aussi en Italie la parution de l'ouvrage de G. Rametta, La metafisica di Bradley e la sua recezione nel pensiero del primo Novecento, CLEUP, 2006. Sur le sujet des rapports plutôt inattendus entre la philosophie de Bradley et celle de Wittgenstein, signalons C. Wright, «The Moral Organism », dans A. Manser et G. Stock (dir.), The Philosophy of F.H. Bradley, op. cit., p. 77-97.

149. La métaphysique de Collingwood reste encore comparativement peu étudiée. Voir, A. Donagan, The Later Philosophy of R. G. Collingwood, $2^{\mathrm{e}}$ éd., Chicago, University of Chicago Press, 1985; G. D'Oro, Collingwood and the Metaphysics of Experience, Londres, Routledge, 2002, M. Krausz (dir.), Critical Essays on the Philosophy of R. G. Collingwood, Oxford, Clarendon Press, 1972; L. O. Mink, Mind, History, and Dialectic: The Philosophy of R. G. Collingwood, Bloomington, Indiana University Press, 1969; L. Rubinoff, Collingwood and the Reform of Metaphysics: A Study in the Philosophy of Mind, Toronto, University of Toronto Press, 1970.

150. G. D'Oro, Collingwood and the Metaphysics of Experience, op. cit.

151. R. G. Collingwood, The New Leviathan, rev. ed., Oxford, Clarendon Press, 1992; traduction française: Le Nouveau Léviathan, Paris, Kimé, 2001. Sur la philosophie politique de Collingwood: D. Boucher, The Social and Political Thought of R. G. Collingwood, Cambridge, Cambridge University Press, 1989; J. Connelly, Metaphysics, Method and Politics: The Political Philosophy of R.G. Collingwood, Exeter, Imprint Academic, 2003; G. K. Browning, Rethinking R. G. Collingwood. Philosophy, Politics and the Unity of Theory and Practice, Basingstoke, Palgrave MacMillan, 2004.

152. G. Ryle, «Mr Collingwood and the Ontological Argument », dans Collected Papers, Bristol Thoemmes, 1990, vol. 2, p. 101. La correspondance Collingwood-Ryle qui en a résulté est désormais publiée dans R. G. Collingwood, An Essay on Philosophical Method, op. cit., p. 253-326. 
réputation ${ }^{153}$. Par ailleurs, la conception de la métaphysique comme entreprise de description de "constellations de présuppositions absolues ", développée dans An Essay on Metaphysics ${ }^{154}$, qui a pourtant été initialement conçu par Collingwood comme une réplique à la critique de la métaphysique élaborée par Ayer, a très tardivement reçu des éloges de la part de ce dernier ${ }^{155}$; peut-être est-ce parce que les présuppositions absolues préfigurent les «propositions charnières » de Wittgenstein et leurs «constellations », les «paradigmes» de Kuhn, ce qui n'a cependant pas empêché Stephen Toulmin de critiquer Collingwood, qu'il rapproche sur cette question du relativisme de Feyerabend ${ }^{156}$.

Avec Outlines of a Philosophy of Art et Principles of Art ${ }^{157}$, Collingwood fut aussi un des rares philosophes britanniques à produire une philosophie de l'art dans la première moitié du vingtième siècle, précédé entre autres par Carritt et Bosanquet ${ }^{158}$. (Pourtant, comme le montre Bill Mander dans sa contribution, "Les idéalistes britanniques et la poésie », les idéalistes entretenaient un rapport privilégié à la poésie, ce qui est entre autres un reflet de l'importance de celle-ci dans une culture victorienne souvent perçue comme étant animée par des valeurs bassement matérialistes.) L'œuvre de Collingwood a été négligée pendant fort longtemps à cause des critiques dévastatrices formulées par Richard Wollheim - auteur, par ailleurs, d'une importante étude sur Bradley ${ }^{159}$ - dans Art and its Objects ${ }^{160}$. On a longtemps confondu la philosophie de l'art de Collingwood avec celle de Croce, ce qui a eu pour effet de la faire apparaître comme archaïque. Or la théorie de Collingwood préfigure entre autres celle de... Wollheim sur la critique d'art comme « réappropriation " $(\text { retrieval })^{161}$, mise en avant dans Art and its Objects! L'article de Chinatsu Kobayashi montre en effet que Collingwood a suivi Bosanquet dans sa critique de Croce et que sa philosophie de l'art ne peut donc nullement

153. G. Ryle, «Philosophical Arguments», dans Collected Papers, op. cit., vol. 2, p. 195.

154. R. G. Collingwood, An Essay on Metaphysics, Oxford, Clarendon Press, 1940, $2^{\mathrm{e}}$ éd., 1998, chapitres VI-V.

155. A. J. Ayer, Philosophy in the Twentieth Century, Londres, Orion, 1992, chapitre VII.

156. Voir, par exemple, S. Toulmin, "Conceptual Change and the Problem of Relativity ", dans M. Krausz (dir.) Critical Essays on the Philosophy of R. G. Collingwood, Oxford, Clarendon Press, 1972, p. 201-221.

157. R. G. Collingwood, Outlines of a Philosophy of Art, op. cit. et The Principles of Art, Oxford, Clarendon Press, 1938.

158. B. Bosanquet, A History of Aesthetics, Londres, Swan Sonnenschein \& Co., 1892; E. F. Carritt, What is Beauty? A First Introduction to the Subject and to Modern Theories, Oxford, Clarendon Press, 1932, et An Introduction to Aesthetics, Londres, Hutchinson, 1949. Outre les idéalistes, seul le réaliste Samuel Alexander a publié plusieurs ouvrages d'esthétique à cette époque, mais il ne faut pas oublier les travaux très influents des critiques d'art de cette époque, comme Clive Bell, Roger Fry et Herbert Read.

159. R. Wollheim, F. H. Bradley, Harmondsworth, Penguin Books, 1959.

160. R. Wollheim, Art and its Objects, $2^{\mathrm{e} e}$ é., Cambridge, Cambridge University Press, 1980 ; traduction française : L'art et ses objets, Paris, Aubier, 1994.

161. Ibidem, p. 185-204; traduction française, p. 169-185. 
être confondue avec celle de l'Italien; elle est en effet proche de celle de Wollheim. Les récents travaux d'Aaron Ridley ont par ailleurs ouvert bien des yeux sur la philosophie de l'art de Collingwood ${ }^{162}$. Un des débats les plus importants des dernières années en esthétique analytique porte sur la réappropriation par le spectateur de l'intention de l'artiste dans l'interprétation de l'œuvre d'art, et on peut espérer que les idées de Collingwood y jouent un rôle.

Bien sûr, une relecture des œuvres des idéalistes, libre des préjugés autrefois entretenus à leur égard, n'implique pas que nous acceptions leurs conclusions ou que nous n'examinions pas avec rigueur la validité et la portée réelle de leurs arguments. Le style de certains, comme Bradley, pourra paraître rébarbatif, tandis que d'autres, comme Bosanquet, apparaîtront comme n'étant pas assez rigoureux. Leurs préoccupations aussi ne sont plus les nôtres on pense entre autres au lien étroit avec la pensée religieuse -, mais il semble désormais clair, malgré tout cela, que de nombreuses discussions contemporaines peuvent être enrichies par une meilleure connaissance de leurs œuvres. Les contributions à ce numéro, de provenance «analytique» ou non, sont donc une invitation à une lecture sans préjugés, pour qui veut chercher les bons arguments là où ils se trouvent ${ }^{163}$.

162. Voir A. Ridley, «Not Ideal: Collingwood's Expression Theory ", Journal of Aesthetics and Art Criticism, vol. 55 (1997), p. 263-72 et R. G. Collingwood: A Philosophy of Art, Londres, Phoenix, 1998. Voir aussi de David Davies, "Collingwood's 'Performance' Theory of Art », British Journal of Aesthetics, vol. 48, 2008, p. 162-174.

163. Nous aimerions remercier Leslie Armour, James Bradley, Mathias Girel, Jérôme Havenel, Laurent Jaffro, François Latraverse, Bill Mander et Gaetano Rametta et William Sweet pour leur aide précieuse. 